\title{
ALMOST CYCLIC ELEMENTS IN CROSS-CHARACTERISTIC REPRESENTATIONS OF FINITE GROUPS OF LIE TYPE
}

\author{
LINO DI MARTINO, MARCO A. PELLEGRINI, AND ALEXANDRE E. ZALESSKI
}

\begin{abstract}
This paper is a significant contribution to a general programme aimed to classify all projective irreducible representations of finite simple groups over an algebraically closed field, in which the image of at least one element is represented by an almost cyclic matrix (that is, a square matrix $M$ of size $n$ over a field $\mathbb{F}$ with the property that there exists $\alpha \in \mathbb{F}$ such that $M$ is similar to $\operatorname{diag}\left(\alpha \cdot \operatorname{Id}_{k}, M_{1}\right)$, where $M_{1}$ is cyclic and $\left.0 \leq k \leq n\right)$. While a previous paper dealt with the Weil representations of finite classical groups, which play a key role in the general picture, the present paper provides a conclusive answer for all cross-characteristic projective irreducible representations of the finite quasi-simple groups of Lie type and their automorphism groups.
\end{abstract}

\section{INTRODUCTION}

Let $V$ be a vector space of finite dimension $n$ over an arbitrary field $\mathbb{F}$, and let $M$ be a square matrix of size $n$ over $\mathbb{F}$. Then $M$ is said to be cyclic if the characteristic polynomial and the minimum polynomial of $M$ coincide. Note that a matrix $M \in \operatorname{Mat}_{n}(\mathbb{F})$ is cyclic if and only if the $\mathbb{F}\langle M\rangle$-module $V$ is cyclic, that is, is generated by a single element. This is standard terminology in module theory, and the source of the term 'cyclic matrix'. Matrices with simple spectrum often arising in applications are cyclic. We consider a generalisation of the notion of cyclic matrix, namely, we define a matrix $M \in \operatorname{Mat}_{n}(\mathbb{F})$ to be almost cyclic if there exists $\alpha \in \mathbb{F}$ such that $M$ is similar to $\operatorname{diag}\left(\alpha \cdot \operatorname{Id}_{k}, M_{1}\right)$, where $M_{1}$ is cyclic and $0 \leq k \leq n$.

Examples of almost cyclic matrices arise naturally in the study of matrix groups over finite fields. For instance, if an element $g \in \mathrm{GL}(V)$ acts irreducibly on $V / V^{\prime}$, where $V^{\prime}$ is some eigenspace of $g$ on $V$, then $g$ is almost cyclic. Transvections and reflections are other examples, as well as unipotent matrices with Jordan form consisting of a single non-trivial block.

The aim of the present paper is to implement a crucial step within a general project, which can be stated as follows: determine all irreducible subgroups $G$ of $\mathrm{GL}(V)$ which are generated by almost cyclic matrices, mainly when $\mathbb{F}$ is algebraically closed. The motivations for such a programme (including the connections with the area of linear group recognition) have been described in full detail in [6], to which paper we also refer for the citations of all relevant past results connected to our subject.

As current applications seem to focus on $p$-elements, we will limit ourselves to the study of elements $g \in G$ of any $p$-power order, $p$ a prime. Furthermore, we

2010 Mathematics Subject Classification. 20C33, 20C15, 20G40.

Key words and phrases. Finite groups of Lie type, Cross-characteristic representations, Almost cyclic matrices, Eigenvalue multiplicities. 
will make a systematic use of representation theory and will take for granted the classification theorem of finite simple groups. In view of it, the non-abelian finite simple groups consist of 26 sporadic groups, the alternating groups of degree $>4$, and the so-called finite groups of Lie type. The latter come together with their defining characteristics, and in this paper it will be convenient for us to distinguish two groups of Lie type of distinct defining characteristic even if they are isomorphic. For instance, we view $\mathrm{PSL}_{3}(2)$ and $\mathrm{PSL}_{2}(7)$ as distinct.

The sporadic simple groups and their covering groups have been completely dealt with in [3. In [5], we started to deal with finite groups of Lie type, and determined all the irreducible representations of a quasi-simple group of Lie type $G$ over an algebraically closed field $\mathbb{F}$ of characteristic coprime to the defining characteristic of $G$, in which the image of at least one unipotent element $g$ is represented by an almost cyclic matrix. The complementary case, when $g$ is unipotent in $G$, and the characteristic of $\mathbb{F}$ is the defining characteristic of $G$, has been settled for classical groups in 27], and for the exceptional groups of Lie type in [29]. This leaves open the case when $g$ is a semisimple element of prime-power order of $G$. The case where $g^{2}=1$ already attracted attention many years ago, and was treated in 25, 32, 36, 37.

We should also mention that the occurrence of almost cyclic $p$-elements in irreducible $\mathbb{F}$-representation of finite simple groups, where $p=\ell=\operatorname{char} \mathbb{F}$, has been studied recently in [2].

In [6], the focus was on Weil representations of finite classical groups (there, an ample overview of these representations is given in Section 5.1). The reason to deal first with Weil representations was the strong evidence that most examples of semisimple almost cyclic elements occur in Weil representations, and the actual occurrence of such elements was thoroughly examined in [․

In the present paper, we will consider the occurrence of almost cyclic elements of prime-power order in projective cross-characteristic irreducible representations $\phi$ of finite simple classical groups $L$ which are not Weil (Sections 5, 6, 7). Furthermore, we will also consider the group extensions $G$ of groups $L$ by outer automorphisms (note that a separate ad hoc section (Section 4) is required for the case when $\left.\operatorname{PSL}_{2}(q) \subseteq G \subseteq \operatorname{AutPSL}_{2}(q)\right)$. Finally, we will also deal with the projective irreducible representations of finite simple exceptional groups of Lie type and their automorphism groups (Section 8).

We note explicitly that, for the sake of completeness, we choose to consider as simple groups of Lie type the commutator subgroups of the groups $\operatorname{PSp}_{4}(2), G_{2}(2)$, ${ }^{2} F_{4}(2)$ and ${ }^{2} G_{2}(3)$. The main result of the present paper is summarised in the following:

Main Theorem. Let $L$ be a finite simple group of Lie type, let $g \in$ Aut $L$ be a p-element for some prime $p$, and let $G=\langle L, g\rangle$. Let $\phi$ be an irreducible projective representation of $G$ over an algebraically closed field $\mathbb{F}$ of characteristic $\ell$, different from the defining characteristic of $L$. Suppose that $\phi$ is non-trivial on $L$; furthermore, assume that $g^{2} \neq 1, L \neq \mathrm{PSL}_{2}(q)$ and $\phi(g)$ is almost cyclic. Then either $\phi_{\mid L}$ is an irreducible Weil representation of $L$, where $L \in\left\{\operatorname{PSL}_{n}(q)(n \geq\right.$ $3), \operatorname{PSU}_{n}(q)(n \geq 3), \operatorname{PSp}_{2 n}(q)(n \geq 2, q$ odd $\left.)\right\}$ and $g$ is semisimple, or $L$ belongs to the following list of groups:

$$
\begin{aligned}
\mathcal{L}= & \left\{\mathrm{PSL}_{3}(2), \mathrm{PSL}_{3}(4), \mathrm{PSL}_{4}(2), \mathrm{PSU}_{3}(3), \mathrm{PSU}_{3}(4), \mathrm{PSU}_{4}(2), \mathrm{PSp}_{4}(3), \mathrm{PSU}_{5}(2),\right. \\
& \left.\mathrm{PSp}_{4}(2)^{\prime}, \mathrm{PSp}_{4}(4), \mathrm{PSp}_{6}(2), \mathrm{P}_{8}^{+}(2),{ }^{2} B_{2}\left(2^{3}\right), G_{2}(2)^{\prime}, G_{2}(3), G_{2}(4),{ }^{2} G_{2}(3)^{\prime}\right\} .
\end{aligned}
$$


A more detailed statement, providing full information on the groups $L$ belonging to $\mathcal{L}$, is given in Theorem 9.1. The case when $g$ is an involution is fully dealt with in Lemma 2.10 for classical groups, and in Lemma 8.1 for the groups of exceptional type.

One of our main tools is Lemma 2.4, which gives, for an arbitrary finite group $G$, an upper bound for $\operatorname{dim} \phi$ in terms of the order of $g \in G$, provided $g$ is almost cyclic. For many pairs $(\phi, g)$ this upper bound conflicts with the known lower bound for $\operatorname{dim} \phi$, which violates the existence of the pair in question.

In order to apply that lemma to a group $G$, where $L \subseteq G \subseteq$ Aut $L$, and $L$ is a simple group of Lie type, we need an accurate upper bound for $\eta_{p}($ Aut $L)$, the exponent of a Sylow $p$-subgroup of Aut $L$, and for $\alpha(g)$, the minimal number of conjugates of $g$ generating $G$. Results on $\eta_{p}$ (Aut $L$ ) are collected in Section 3 . Rather precise information on $\alpha(g)$ is available in a paper by Guralnick and Saxl 14, which is our main reference for this matter. If $g \in L$ and $C_{L}(g)$ contains no unipotent elements, then $\alpha(g) \leq 3$ (see Lemma 2.7, deduced from a result of Gow [11). However, if $\operatorname{dim} \phi$ is relatively small in comparison with $\eta_{p}(G)$, much more analysis is required. This actually happens either if $|L|$ is small or if $G=L$ and $\phi$ is a Weil representation. (As already mentioned, the Weil representations have been dealt with in 6 ]).

In our analysis we will deal separately with the following cases: (i) $|g|=2$ (see Lemma 2.10 and Section 8); (ii) $L=\operatorname{PSL}_{2}(q)$ (see Section 4); (iii) The groups $\operatorname{PSL}_{n}(q), n>2 ; \operatorname{PSU}_{n}(q), n>2 ; \operatorname{PSp}_{2 n}(q), n>1, q$ odd (see Section 5); (iv) Some exceptional low-dimensional classical groups (see Section 6); (v) The groups $\operatorname{Sp}_{2 n}(q), n>1, q$ even; $\Omega_{2 n+1}(q), n>2, q$ odd; $\mathrm{P} \Omega_{2 n}^{ \pm}(q), n>3$ (see Section 7); (vi) The exceptional groups of Lie type (see Section 8 ).

Notation. The centre of a group $G$ is denoted by $Z(G)$. Let $G$ be a finite group. We write $|G|$ for the order of $G$. For $g \in G,|g|$ is the order of $g$, and $o(g)$ is the order of $g$ modulo $Z(G)$, that is, the minimum integer $k$ such that $g^{k} \in Z(G)$. If $g$ does not lie in a proper normal subgroup of $G$, we write $\alpha(g)$ for the minimum number of conjugates of $g$ that generate $G$. For a prime $p$ we denote by $\eta_{p}(G)$ the exponent of a Sylow $p$-subgroup of $G$.

A finite field of order $q$ (where $q$ is a prime-power) is denoted by $\mathbb{F}_{q}$. Let $V$ be a vector space of finite dimension $m>1$ over the finite field $\mathbb{F}_{q}$. The general linear group $\mathrm{GL}(V)$, that is, the group of all bijective linear transformations of $V$, and the special linear group $\mathrm{SL}(V)$, that is, the subgroup of $\mathrm{GL}(V)$ consisting of the transformations of determinant 1 , are denoted by $\mathrm{GL}_{m}(q)$ and $\mathrm{SL}_{m}(q)$, respectively.

Suppose that the space $V$ is endowed with a non-degenerate orthogonal, symplectic or unitary form. Then $I(V)$ denotes the group of the isometries of $V$, that is the set of all elements $x \in \mathrm{GL}(V)$ preserving the form. Then we will loosely use the term 'finite classical group' for a subgroup $G$ of $I(V)$ containing the commutator subgroup $I(V)^{\prime}$, as well as the projective image of $G$, that is, the quotient of $G$ modulo its scalars, which will be denoted by $\mathrm{P} G$. In particular: if $V$ is a symplectic space over $\mathbb{F}_{q}, I(V)$ will be denoted by $\operatorname{Sp}_{m}(q)$; if $V$ is a unitary space over the field $\mathbb{F}_{q^{2}}, I(V)$ will be denoted by $\mathrm{GU}_{m}(q)$; and if $V$ is an orthogonal space over $\mathbb{F}_{q}, I(V)$ will be denoted by $\mathrm{O}_{m}(q)$. More precisely, if $V$ is an orthogonal space of even dimension $m$, we will denote $I(V)$ by $\mathrm{O}_{m}^{+}(q)$ or $\mathrm{O}_{m}^{-}(q)$ if $V$ has maximal or non-maximal Witt index, respectively. Accordingly, the subgroups of the unitary and orthogonal isometry groups consisting of the elements of determinant 1 
will be denoted by $\mathrm{SU}_{m}(q), \mathrm{SO}_{m}(q), \mathrm{SO}_{m}^{+}(q)$ and $\mathrm{SO}_{m}^{-}(q)$, respectively. We recall that $\operatorname{Sp}_{m}(q)^{\prime}=\operatorname{Sp}_{m}(q)$, unless $m=2$ and $q \leq 3$, or $(m, q)=(4,2)$, whereas $\mathrm{GU}_{m}(q)^{\prime}=\mathrm{SU}_{m}(q)$, unless $m=2$ and $q \leq 3$, or $(m, q)=(3,2)$. In the orthogonal case, the groups $\mathrm{SO}_{m}(q), \mathrm{SO}_{m}^{+}(q)$ and $\mathrm{SO}_{m}^{-}(q)$ contain $I(V)^{\prime}$ as a subgroup of index 2. We will denote by $\Omega_{m}(q)$ and $\Omega_{m}^{ \pm}(q)$ the commutator subgroups of the corresponding orthogonal groups, and accordingly, we will refer to a classical group as a subgroup of $I(V)$ containing the commutator subgroup $I(V)^{\prime}$. Furthermore, we will denote by $\operatorname{CSp}_{2 n}(q)$ the conformal symplectic group, that is, the group of symplectic similarities. Also, it should be noted that in places the term 'classical group' will be meant to include also the groups $\mathrm{GL}_{m}(q)$ and $\mathrm{SL}_{m}(q)$ (considering $V$ endowed with the identically zero bilinear form). It is well-known that, for $m>2$, or $m=2$ and $q>2$, one has $\mathrm{GL}_{m}(q)^{\prime}=\mathrm{SL}_{m}(q)$.

If $G$ is a finite group of Lie type of defining characteristic $r$, we assume throughout the paper that $\ell$ is coprime to $r$. Furthermore, note that: (1) the conjugacy classes of elements of $G$ are labelled according to the GAP package [8]; (2) the extensions $G$ of a finite simple group of Lie type $L$ by an outer automorphism will be labelled according to the Atlas notation as given in [1] and [20, unless we wish to indicate explicitly that $G$ is the extension of $L$ by the group of field automorphisms, in which case $G$ will be denoted, say, by $\mathrm{P}^{2} \mathrm{~L}_{m}(q)$ or $\mathrm{P} \mathrm{U}_{m}(q)$ for $L=\operatorname{PSL}_{m}(q)$ or $L=\operatorname{PSU}_{m}(q)$, respectively.

By a projective $\mathbb{F}$-representation of an arbitrary group $G$ we mean a homomorphism $\phi: G \rightarrow \mathrm{PGL}_{m}(\mathbb{F})$ for some $m$. Let $\bar{G}$ denote the pre-image of $\phi(G)$ in $\mathrm{GL}_{m}(\mathbb{F})$ and let $\psi$ denote the linear representation of $\bar{G}$ arising in this way from $\phi$. If $g \in G$, and $\bar{g}$ denotes a pre-image of $\phi(g)$ in $\mathrm{GL}_{m}(\mathbb{F})$, we will say for short that $\phi(g)$ is almost cyclic if so is $\psi(\bar{g})$ in $\mathrm{GL}_{m}(\mathbb{F})$. Note that, clearly, the property of $\phi(g)$ of being almost cyclic does not depend on the choice of $\bar{g}$.

\section{Preliminaries}

We recall the following basic definition:

Definition 2.1. Let $M$ be an $(n \times n)$-matrix over an arbitrary field $\mathbb{F}$. We say that $M$ is almost cyclic if there exists $\alpha \in \mathbb{F}$ such that $M$ is $\operatorname{similar}$ to $\operatorname{diag}\left(\alpha \cdot \operatorname{Id}_{k}, M_{1}\right)$, where $M_{1}$ is cyclic and $0 \leq k \leq n$.

It is trivial to observe that every $(3 \times 3)$-matrix over any field $\mathbb{F}$ is almost cyclic. Another elementary observation, which will be useful throughout the paper, is the following: if $M \in \mathrm{GL}(V)$ is almost cyclic, and $U$ is an $M$-stable subspace of $V$, then the induced actions of $M$ on $U$ and on $V / U$ yield almost cyclic matrices.

Throughout the paper, unless stated otherwise, we assume that $\mathbb{F}$ is an algebraically closed field of characteristic $\ell$. We emphasise that the representations of $G$ we will consider are all over $\mathbb{F}$.

2.1. Almost cyclicity and dimension bounds. Let $G$ be a finite group and $\phi$ be a projective irreducible $\mathbb{F}$-representation of $G$. Let $g \in G$ and suppose that $\phi(g)$ is almost cyclic and non-scalar. Our results will be essentially based on the fact that $\operatorname{dim} \phi$ can be bounded in terms of the minimum number $\alpha(g)$ of conjugates of $g$ sufficient to generate $G$. In this subsection we explain this in detail.

The following crucial results on generation by conjugates are due to Guralnick and Saxl [14]. 
Proposition 2.2. Let $L$ be a simple group of Lie type, and let $1 \neq x \in$ Aut $L$. Denote by $\alpha(x)$ the minimum number of $L$-conjugates of $x$ sufficient to generate $\langle x, L\rangle$. Then the following holds:

(1) 14, Theorem 4.2] Let $L$ be a simple classical group, and assume that the natural module for $L$ has dimension $n \geq 5$. Then $\alpha(x) \leq n$, unless $L=$ $\operatorname{PSp}_{n}(q)$ with $q$ even, $x$ is a transvection and $\alpha(x)=n+1$.

(2) [14, Theorem 5.1] Let $L$ be a simple exceptional group of Lie type, of untwisted Lie rank $m$. Then $\alpha(x) \leq m+3$, except possibly for the case $L=F_{4}(q)$ with $x$ an involution, where $\alpha(x) \leq 8$.

Proposition 2.3. Under the same assumptions and of Proposition 2.2, and assuming additionally that $x$ has prime order, the following holds:

(1) [14, Lemma 3.1] Let $L=\operatorname{PSL}_{2}(q)$, with $q \geq 4$. Then $\alpha(x) \leq 3$, unless either (i) $x$ is a field automorphism of order 2 and $\alpha(x) \leq 4$, except that $\alpha(x)=5$ for $q=9$; or (ii) $q=5, x$ is a diagonal automorphism of order 2 and $\alpha(x)=4$. Moreover, if $x$ has odd order, then $\alpha(x)=2$, unless $q=9$, $|x|=3$ and $\alpha(x)=3$.

(2) [14, Lemma 3.2] If $L=\operatorname{PSL}_{3}(q)$, then $\alpha(x) \leq 3$ unless $x$ is an involutory graph-field automorphism and $\alpha(x) \leq 4$.

(3) [14, Lemma 3.3] If $L=\operatorname{PSU}_{3}(q), q>2$, then $\alpha(x) \leq 3$, unless $q=3$ and $x$ is an inner involution with $\alpha(x)=4$.

(4) [14, Theorem 4.1(c),(d)] If $L=\operatorname{PSL}_{4}(q)$, then $\alpha(x) \leq 4$, unless one of the following holds: (i) $q>2, x$ is an involutory graph automorphism and $\alpha(x) \leq 6$; (ii) $q=2, x$ is an involutory graph automorphism and $\alpha(x) \leq 7$.

(5) [14, Lemma 3.4] If $L=\mathrm{PSU}_{4}(q)$, then $\alpha(x) \leq 4$, unless one of the following holds: (i) $x$ is an involutory graph automorphism and $\alpha(x) \leq 6$; (ii) $q=2$ with $x$ a transvection and $\alpha(x) \leq 5$.

(6) [14, Theorem 4.1(f)] If $L=\mathrm{PSp}_{4}(q)$, then $\alpha(x) \leq 4$, unless $x$ is an involution and $\alpha(x) \leq 5$, or $q=3$ and $\alpha(x) \leq 6$.

The above quoted bounds on $\alpha(x)$ play a role in the following lemma, which is crucial for our purposes.

Lemma 2.4. Let $H$ be a finite group, let $\phi: H \rightarrow \mathrm{PGL}_{m}(\mathbb{F})$ be an irreducible projective $\mathbb{F}$-representation of $H$, where $\mathbb{F}$ is an arbitrary algebraically closed field, and let $\bar{H}$ be the pre-image of $\phi(H)$ in $\mathrm{GL}_{m}(\mathbb{F})$. Let $M$ be the module affording the linear representation $\psi$ of $\bar{H}$ arising from $\phi$. For any $h \in H$ denote by $\bar{h}$ a preimage of $\phi(h)$ in $\bar{H}$, and choose $h \in H$ such that $H$ is generated some conjugates of $h$. Let $d=\operatorname{dim}(\lambda \cdot \operatorname{Id}-\bar{h}) M \neq 0$ for some $\lambda \in F$. Then $\operatorname{dim} \phi \leq d \alpha(h)$. In particular, if $\phi(h)$ is almost cyclic, then $\operatorname{dim} \phi \leq \alpha(h)(o(h)-1) \leq \alpha(h)(|h|-1)$.

Proof. Let $m=\alpha(h)$ and $h_{1}, \ldots, h_{m}$ be conjugates of $h$ generating $H$. Let $M_{i}=$ $\left(\lambda \cdot \operatorname{Id}-h_{i}\right) M$. Then every $\bar{h}_{i}$ acts scalarly as $\lambda$ on $M / M_{i}$, and hence it acts scalarly as $\lambda$ on any quotient $M / U$ where $U$ is a subspace containing $M_{i}$, thus in particular on $M /\left(\sum M_{i}\right)$. This is true for every $i$, so every $\bar{h}_{i}$ acts as $\lambda$ on $M /\left(\sum M_{i}\right)$. Since $\left\langle h_{1}, \ldots, h_{m}\right\rangle=H$, the group $\bar{H}$ acts scalarly on $M /\left(\sum M_{i}\right)$. It follows that $\sum M_{i}$ is a non-zero $\bar{H}$-submodule of $M$, and hence coincides with $M$. Since $\operatorname{dim}\left(\sum_{i=1}^{m}(\lambda\right.$. $\left.\left.\operatorname{Id}-\bar{h}_{i}\right) M\right) \leq \sum_{i=1}^{m} \operatorname{dim}\left(\lambda \cdot \operatorname{Id}-\bar{h}_{i}\right) M=m \cdot \operatorname{dim}(\lambda \cdot \operatorname{Id}-\bar{h}) M=m d$, the first part of the statement follows. The second part is proven in [3, Lemma 2.1]. 
We remark here that throughout the paper we will frequently make use of Lemma 2.4 without explicit reference to it. The following results concerning regular semisimple elements will be useful for our purposes.

Lemma 2.5. 11 Let $G$ be a simple group of Lie type of characteristic $\ell$ and let $1 \neq g \in G$ be a regular semisimple element. Then every semisimple element of $G$ can be expressed as a product $a b$, where $a, b$ are conjugate to $g$.

As a corollary of Lemma 2.5. we obtain a small refinement of case (1) of Proposition 2.3 when considering 2-elements:

Lemma 2.6. Let $L=\mathrm{PSL}_{2}(q)$, where $q$ is odd and $q>9$. Let $g \in L$ be a 2-element such that $g^{2} \neq 1$. Then $\alpha(g)=2$.

Proof. Note that $g$ is a regular semisimple element of $L$ (as $C_{L}(g)$ contains no unipotent element). So, by Lemma 2.5, there exists $x \in L$ conjugate to $g$ such that $g x=s$, where $s$ is a Singer cycle of $L$. If $q>9$, it is well-known that every proper subgroup of $L$ containing $s$ is either $S=\langle s\rangle$ or $N=N_{L}(S)$, a dihedral group of order $2|S|$. Therefore, the result is true unless both $g, x \in N$. But the latter is not the case. Indeed, suppose that $g, x \in N$. As $N$ is dihedral, all the 2-elements of order $\geq 4$ of $N$ are in $S$. Therefore $g \in S$ and $x \in S$. As $S$ is cyclic and $|g|=|x|$, it follows readily that $|g x|<|g|$; this obviously contradicts $g x=s$, as claimed.

Lemma 2.7. 6, Lemma 2.8] Let $G$ be a simple group of Lie type. Then the following holds:

(1) $G$ is generated by two semisimple elements.

(2) Let $g \in G$ be any regular semisimple element. Then $\alpha(g) \leq 3$, that is, $G$ can be generated by three elements conjugate to $g$.

The following two lemmas are crucial for us in view of the results above. The first one yields the best known lower bounds for the dimension of an irreducible projective cross-characteristic $\mathbb{F}$-representation of a finite classical group. The second one provides lower bounds for the dimension of any non-Weil irreducible projective cross-characteristic $\mathbb{F}$-representation of a finite classical group.

Lemma 2.8. 19, Table 1] Let $L$ be a simple classical group and let $\phi$ be an irreducible projective cross-characteristic $\mathbb{F}$-representation of $L$, with $\operatorname{dim} \phi>1$. Then the following holds:

(1) If $L=\mathrm{PSL}_{2}(q)$, then $\operatorname{dim} \phi \geq \frac{q-1}{(2, q-1)}$, unless $q=4,9$; in the latter exceptional cases, $\operatorname{dim} \phi \geq 2,3$, respectively.

(2) If $L=\operatorname{PSL}_{n}(q), n>2$, then $\operatorname{dim} \phi \geq \frac{q^{n}-1}{q-1}-2$, unless $(n, q)=(3,2),(3,4)$, $(4,2),(4,3)$; in the latter exceptional cases, $\operatorname{dim} \phi \geq 2,4,7,26$, respectively.

(3) If $L=\operatorname{PSU}_{n}(q), n>2,(n, q) \neq(4,2),(4,3)$, then $\operatorname{dim} \phi \geq \frac{q^{n}-q}{q+1}$ if $n$ is odd, while $\operatorname{dim} \phi \geq \frac{q^{n}-1}{q+1}$ if $n$ is even; if $(n, q)=(4,2),(4,3)$, then $\operatorname{dim} \phi \geq 4,6$, respectively.

(4) If $L=\operatorname{PSp}_{2 n}(q), n>1$, and $q$ is odd, then $\operatorname{dim} \phi \geq \frac{q^{n}-1}{2}$.

(5) If $L=\operatorname{PSp}_{2 n}(q)$, where $n>1, q$ is even, and $(n, q) \neq(4,2)$, then $\operatorname{dim} \phi \geq$ $\frac{q\left(q^{n}-1\right)\left(q^{n-1}-1\right)}{2(q+1)}$. If $L=\operatorname{PSp}_{4}(2)^{\prime}$, then $\operatorname{dim} \phi \geq 2$.

(6) Let $L=\mathrm{P} \Omega_{2 n+1}(q)$, where $n>2$ and $q$ is odd. If $q=3$, and $n \neq 3$, then $\operatorname{dim} \phi \geq \frac{\left(3^{n}-1\right)\left(3^{n}-3\right)}{3^{2}-1}$; if $q>3$, then $\operatorname{dim} \phi \geq \frac{q^{2 n}-1}{q^{2}-1}-2$; if $(n, q)=(3,3)$, then $\operatorname{dim} \phi \geq 27$. 
(7) Let $L=\mathrm{P} \Omega_{2 n}^{+}(q), n>3$. If $q<4$ and $(n, q) \neq(4,2)$, then $\operatorname{dim} \phi \geq$ $\frac{\left(q^{n}-1\right)\left(q^{n-1}-1\right)}{q^{2}-1}$; if $q \geq 4$, then $\operatorname{dim} \phi \geq \frac{\left(q^{n}-1\right)\left(q^{n-1}+q\right)}{q^{2}-1}-2$; if $L=\mathrm{P} \Omega_{8}^{+}(2)$, then $\operatorname{dim} \phi \geq 8$.

(8) Let $L=\mathrm{P} \Omega_{2 n}^{-}(q), n>3$, where $(n, q) \neq(4,2),(4,4),(5,2),(5,3)$. Then $\operatorname{dim} \phi \geq \frac{q\left(q^{n}+1\right)\left(q^{n-2}-1\right)}{q^{2}-1}-1 ;$ for the exceptional cases, $\operatorname{dim} \phi \geq 32,1026,151$, 2376 , respectively.

From [13, Theorems 2.1 and 2.7], [15, Theorem 1.1], [16] and [18, Theorem 1.6] we obtain the following:

Lemma 2.9. Let $L \in\left\{\mathrm{PSL}_{n}(q), n \geq 3 ; \operatorname{PSU}_{n}(q), n \geq 3 ; \operatorname{PSp}_{2 n}(q), n \geq 2, q\right.$ odd $\}$ and let $\phi$ be a non-trivial cross-characteristic projective irreducible representation of $L$. Then either $\phi$ is a Weil representation, or one of the following occurs.

(A) If $L=\operatorname{PSL}_{n}(q)$ and $(n, q) \neq(3,2),(3,4),(4,2),(4,3),(6,2),(6,3)$, then $\operatorname{dim} \phi \geq \vartheta_{n}^{+}(q)$, where

$$
\vartheta_{n}^{+}(q)= \begin{cases}(q-1)\left(q^{2}-1\right) / \operatorname{gcd}(3, q-1) & \text { if } n=3, \\ (q-1)\left(q^{3}-1\right) / \operatorname{gcd}(2, q-1) & \text { if } n=4, \\ \left(q^{n-1}-1\right)\left(\left(q^{n-2}-q\right) /(q-1)-\kappa_{n-2}\right) & \text { if } n \geq 5 .\end{cases}
$$

Here, $\kappa_{n}=1$ if $\ell$ divides $\left(q^{n}-1\right) /(q-1)$ and 0 otherwise. Furthermore, if $(n, q)=(4,3),(6,2),(6,3)$, then $\operatorname{dim} \phi \geq \vartheta_{n}^{+}(q)$, where $\vartheta_{n}^{+}(q)=26,61,362$, respectively.

$\left(B_{1}\right)$ If $L=\operatorname{PSU}_{n}(q)$, with $n \geq 5$ and $(n, q) \neq(6,2)$, then $\operatorname{dim} \phi \geq \vartheta_{n}^{-}(q)$, where

$$
\vartheta_{n}^{-}(q)= \begin{cases}\frac{q^{n-2}(q-1)\left(q^{n-2}-q\right)}{q+1} & \text { if } n \text { is odd, } \\ \frac{q^{n-2}(q-1)\left(q^{n-2}-1\right)}{q+1} & \text { if } n \text { is even. }\end{cases}
$$

$\left(B_{2}\right)$ If $L=\mathrm{PSU}_{4}(q)$ and $q>3$, then $\operatorname{dim} \phi \geq \vartheta_{4}^{-}(q)$, where

$$
\vartheta_{4}^{-}(q)= \begin{cases}\frac{\left(q^{2}+1\right)\left(q^{2}-q+1\right)-2}{2} & \text { if } q \text { is odd, } \\ \left(q^{2}+1\right)\left(q^{2}-q+1\right)-1 & \text { if } q \text { is even. }\end{cases}
$$

$\left(B_{3}\right)$ If $L=\operatorname{PSU}_{3}(q)$ and $q \geq 5$, then $\operatorname{dim} \phi \geq \vartheta_{3}^{-}(q)$, where

$$
\vartheta_{3}^{-}(q)= \begin{cases}\frac{(q-1)\left(q^{2}+3 q+2\right)}{6} & \text { if } \operatorname{gcd}(3, q+1)=3, \\ \frac{2 q^{3}-q^{2}+2 q-3}{3} & \text { if } \operatorname{gcd}(3, q+1)=1 .\end{cases}
$$

(C) If $L=\operatorname{PSp}_{2 n}(q)$ and $(2 n, q) \neq(6,2)$, then $\operatorname{dim} \phi \geq \sigma_{n}(q)$, where

$$
\sigma_{n}(q)=\frac{\left(q^{n}-1\right)\left(q^{n}-q\right)}{2(q+1)} .
$$

2.2. The case $|g|=2$. In this subsection we consider the case $|g|=2$, which yields a significant part of the examples in which almost cyclic elements do occur.

Lemma 2.10. Let $L$ be a finite simple classical group and let $G=\langle L, g\rangle$, where $g \in$ Aut $L$ is an involution. Let $\phi$ be a cross-characteristic projective irreducible $\mathbb{F}$-representation of $G$ which is non-trivial on $L$. Then $\phi(g)$ is almost cyclic if and only if one of the following holds:

(1a) $G=\mathrm{PSL}_{2}$ (4), $\operatorname{dim} \phi=2,3$ and $\ell \neq 2$;

(1b) $G=\mathrm{P} \Sigma \mathrm{L}_{2}$ (4) and either $\operatorname{dim} \phi=4$ and $\ell \neq 2,5$ or $\operatorname{dim} \phi=2,3$ and $\ell=5$;

(2a) $G=\mathrm{PSL}_{2}(5), \operatorname{dim} \phi=2,3$ and $\ell \neq 5$; 
(2b) $G=\mathrm{PGL}_{2}(5), \operatorname{dim} \phi=4$ and $\ell \neq 5$;

(3) $G=\mathrm{PSL}_{2}(7), \operatorname{dim} \phi=3$ and $\ell \neq 7$;

(4a) $G=\mathrm{PSL}_{2}(9), \operatorname{dim} \phi=3$ and $\ell \neq 2,3$;

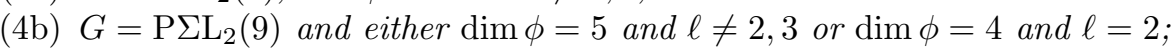

(5) $G=\mathrm{PSL}_{3}(2)$ and either $\operatorname{dim} \phi=3$ and $\ell \neq 2$ or $\operatorname{dim} \phi=2$ and $\ell=7$;

(6) $G=\mathrm{P} \Sigma L_{3}(4), \operatorname{dim} \phi=4$ and $\ell=3$;

(7) $G=\mathrm{PSL}_{4}(2) .2, \operatorname{dim} \phi=7, \ell \neq 2$ and $g$ yields a graph automorphism;

(8a) $G=\mathrm{PSU}_{4}(2), \operatorname{dim} \phi=4,5$ and $\ell \neq 2$ (class $2 a$ );

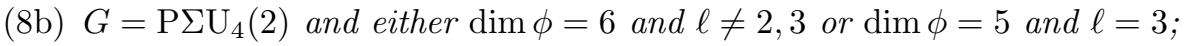

(9) $G=\mathrm{PSU}_{4}(3) \cdot 2_{2}$, $\operatorname{dim} \phi=6$ and $\ell \neq 3$;

(10a) $G=\mathrm{PSp}_{4}(3)$ and either $\operatorname{dim} \phi=4$ and $\ell \neq 3$ (class $2 a$ ) or $\operatorname{dim} \phi=5$ and $\ell \neq 2,3$ (class $2 a$ );

(10b) $G=\mathrm{PCSp}_{4}(3), \operatorname{dim} \phi=6$ and $\ell \neq 2,3$;

(11) $G=\operatorname{PSp}_{6}(2), \operatorname{dim} \phi=7$ and $\ell \neq 2$ (class $\left.2 a\right)$;

(12) $G=\mathrm{PSO}_{8}^{+}(2), \operatorname{dim} \phi=8$ and $\ell \neq 2$.

Proof. (I) Suppose that $\phi(g)$ is almost cyclic. By Lemma 2.4, this implies that

$$
\operatorname{dim} \phi \leq \alpha(g)(|g|-1)=\alpha(g) .
$$

An upper bound for the value of $\alpha$ is given by Propositions 2.2 and 2.3, while a lower bound for the value of $\operatorname{dim} \phi$ is given in Lemma 2.8.

For the sake of brevity, we give full details of the computations yielding the statement of the lemma only in the case where $L=\operatorname{PSL}_{n}(q)$. The other classical groups can be dealt with in a similar way.

Let $L=\mathrm{PSL}_{2}(q)$, with $7 \leq q \neq 9$. Then (2.1) yields $\frac{q-1}{(q-1,2)} \leq 4$, which holds only if $q=7$. If $L \in\left\{\mathrm{PSL}_{2}(4), \mathrm{PSL}_{2}(5)\right\}$, then $\operatorname{dim} \phi \geq 2$ and $\alpha(g)=3$ if $g \in L$, $\alpha(g) \leq 4$ otherwise. If $L=\mathrm{PSL}_{2}(7)$, then $\operatorname{dim} \phi \geq 3$ and $\alpha(g)=3$. If $L=\mathrm{PSL}_{2}(9)$, then $\operatorname{dim} \phi \geq 3$ and $\alpha(g)=3$, unless $g \notin L$, in which case $\alpha(g) \leq 5$.

Let $L=\operatorname{PSL}_{3}(q)$, with $q \neq 2$, 4 . In this case (2.1) yields $q^{2}+q-1 \leq 4$, which is false. If $L=\mathrm{PSL}_{3}(2)$, then $\operatorname{dim} \phi \geq 2$ and $\alpha(g)=3$. If $L=\mathrm{PSL}_{3}(4)$, then $\operatorname{dim} \phi \geq 4$ and $\alpha(g)=3$, unless $g \notin L$, in which case $\alpha(g)=4$.

Let $L=\mathrm{PSL}_{4}(q)$, with $q \geq 4$. Then (2.1) yields $q^{3}+q^{2}+q-1 \leq 6$, which is clearly impossible. If $L=\mathrm{PSL}_{4}(2)$, then $\operatorname{dim} \phi \geq 7$ and $\alpha(g) \leq 6$, unless $g \notin L$, in which case $\alpha(g) \leq 7$. If $L=\mathrm{PSL}_{4}(3)$, then $\operatorname{dim} \phi \geq 26$, whence a contradiction.

Finally, let $L=\operatorname{PSL}_{n}(q)$, with $n \geq 5$. Then (2.1) yields $\frac{q^{n}-1}{q-1}-2 \leq n$, a contradiction.

By the computations above, we readily get items (1a) to (7) of the statement.

(II) Conversely, in all the occurrences listed in the statement of the lemma (items (1) to (12)), there actually exists at least one representation $\phi$ of $G$ and a conjugacy class of involutions $g$ such that $\phi(g)$ is almost cyclic. These $\phi$ 's and these $g$ can be readily determined using [1, 20] and the GAP package.

Remark 2.11. The outer automorphism group of the group $L=\mathrm{PSL}_{2}(9)$ is elementary abelian of order 4 . Let $g$ be an outer automorphism of $L$ of order 2. Then $g$ is either a field automorphism, or a diagonal automorphism, or the

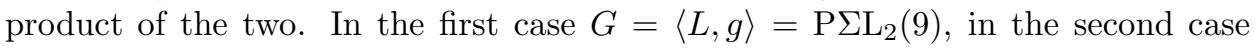
$G=\langle L, g\rangle=\mathrm{PGL}_{2}(9)$, in the third case, $G=\langle L, g\rangle$ is isomorphic to $M_{10}$, a maximal subgroup of the Mathieu group $M_{11}$, a sporadic simple group. It is also well-known that $\mathrm{PSL}_{2}(9) \cong \operatorname{Sp}_{4}(2)^{\prime} \cong \Omega_{4}^{-}(3)$, and $\mathrm{Sp}_{4}(2) \cong \mathrm{P} \Sigma L_{2}(9)$. If we view 
$L$ as $\operatorname{Sp}_{4}(2)^{\prime}$ and let $G=\langle L, g\rangle$, where $g \in$ Aut $L$ is an involution, then the above lemma misses the $\mathbb{F}$-representations of $G$ for $\ell=3$. So, let $\phi$ be a cross-characteristic projective irreducible $\mathbb{F}$-representation of $G$ which is non-trivial on $L$. Then direct inspection shows that $\phi(g)$ is almost cyclic if and only if one of the following occurs:

(1) $\operatorname{dim} \phi=3, G=\operatorname{Sp}_{4}(2)^{\prime}$ and $\ell \neq 2$;

(2) $\operatorname{dim} \phi=3, G=\mathrm{PGL}_{2}(9)$ and $\ell=3$;

(3) $\operatorname{dim} \phi=4, G=\operatorname{Sp}_{4}(2)$, and $\ell=3$;

(4) $\operatorname{dim} \phi=5, G=\operatorname{Sp}_{4}(2)$, and $\ell \neq 2,3$.

\section{Maximum order of a $p$-element in Aut $L$}

Let $L$ be one of the following groups: $\operatorname{PSL}_{n}(q)$, where $n>1$ and $(n, q) \neq$ $(2,2),(2,3) ; \operatorname{PSU}_{n}(q)$, where $n>2$ and $(n, q) \neq(3,2) ; \operatorname{PSp}_{2 n}(q)$, where $n>1$ and $(n, q) \neq(2,2)$. So $L$ is simple. Let $r$ be a prime, and $q=r^{\alpha}$.

Recall that every automorphism of $L$ is a product of inner, diagonal, field and graph automorphisms. Let $A_{d}$ be the subgroup of Aut $L$ generated by all inner and diagonal automorphisms. It is well-known that $A_{d}$ is normal in Aut $L$. Moreover, $A_{d} \cong \mathrm{PGL}_{n}(q)$ or $\mathrm{PGU}_{n}(q)$ if $L=\mathrm{PSL}_{n}(q)$ or $\mathrm{PSU}_{n}(q)$, respectively. Thus, $\left|A_{d} / L\right|=(q-1, n)$ if $L=\operatorname{PSL}_{n}(q)$, and $(q+1, n)$ if $L=\operatorname{PSU}_{n}(q)$. If $L=\operatorname{PSp}_{2 n}(q)$, then $A_{d} \cong \mathrm{PCSp}_{2 n}(q)$. Thus, $\left|A_{d}: L\right|=2$ for $q$ odd, and $A_{d}=L$ for $q$ even. The group Aut $L / A_{d}$ is abelian. Set $\Phi=\operatorname{Gal}\left(\mathbb{F}_{q} / \mathbb{F}_{r}\right)$ and $\Phi_{2}=\operatorname{Gal}\left(\mathbb{F}_{q^{2}} / \mathbb{F}_{r}\right)$. If $L=\operatorname{PSL}_{n}(q)$, then Aut $L / A_{d} \cong C_{2} \times \Phi$, where $C_{2}$ is the cyclic group of order 2 . If $L=\operatorname{PSU}_{n}(q)$, then Aut $L / A_{d} \cong \operatorname{Gal}\left(\mathbb{F}_{q^{2}} / \mathbb{F}_{r}\right)=\Phi_{2}$. If $L=\operatorname{PSp}_{2 n}(q), n>2$, or $n=2$ for $q$ odd, then Aut $L / A_{d} \cong \Phi$. The case $L=\operatorname{PSp}_{4}(q)$ with $q$ even is more complex. In [26, Theorem 28] an automorphism $\phi$ of $\operatorname{Sp}_{4}(q), q$ even, is constructed, such that $\phi^{2}$ is a generator of $\Phi$. It follows that $\operatorname{Aut}_{\mathrm{Sp}_{4}}(q) / \operatorname{Sp}_{4}(q)$ is a cyclic group of order $2|\Phi| \cong \Phi_{2}$. So, if $g \in$ Aut $L$ is a 2-element and $|\Phi|_{2}=2^{m}$, then $g^{2^{m+1}} \in L$. See [23, Table 5.1.A] for details.

Let $p$ be a prime. For any positive integer $m$, we denote by $|m|_{p}$ the $p$-part of $m$, that is the highest power of $p$ dividing $m$. Furthermore, for any positive integer $m$, if $p$ does not divide $m$, then $e=e_{p}(m)$ is defined to be the minimum integer $i>0$ such that $p$ divides $m^{i}-1$ if $p>2$. If $p=2$, then $e_{2}(m)$ is defined to be 1 if $4 \mid(m-1)$ and 2 if $4 \mid(m+1)$. Note that $e \geq 1$, and if $e>1$, then $e<\frac{m^{e}-1}{m-1}$.

Lemma 3.1. Let $p$ be a prime.

(1) $e_{p}(q)=e_{p}\left(q^{p^{k}}\right)$. In particular: if $\left|\mathbb{F}_{q^{p^{k}}}^{\times}\right|_{p}>1$, then $\left|\mathbb{F}_{q}^{\times}\right|_{p}>1$.

(2) Suppose that $p \mid(q-1)$ if $p>2$, and $4 \mid(q-1)$ otherwise. Let $k>0$ be an integer. Then $\left|\mathbb{F}_{q^{p^{k}}}^{\times}\right|_{p}=p^{k} \cdot\left|\mathbb{F}_{q}^{\times}\right|_{p}$, equivalently, $\left|q^{p^{k}}-1\right|_{p}=p^{k}|q-1|_{p}$.

Proof. Fermat's little theorem states that $p$ divides $n^{p}-n$ for any integer $n$, and hence $p \mid(n-1)$ if and only if $p \mid\left(n^{p}-1\right)$. So $p \mid\left(q^{i}-1\right)$ if and only if $p \mid\left(\left(q^{p^{k}}\right)^{i}-1\right)$, whence (1). For (2), see [7, Lemma 7.5].

Recall that, for a group $X$ we denote by $\eta_{p}(X)$ the exponent of a Sylow $p$ subgroup of $X$. The following lemma is well known: for instance, see [24].

Lemma 3.2. Let $G=\mathrm{GL}_{n}(q)$, $p$ a prime dividing $|G|,(p, q)=1$. If $e=e_{p}(q)$, then $\eta_{p}(G)=p^{l} \cdot\left|q^{e}-1\right|_{p}$, where $l$ is such that $p^{l} e \leq n<p^{l+1} e$.

Corollary 3.3. Let $q=q_{0}^{2}$ be odd. Then $\eta_{2}\left(\mathrm{GL}_{n}(q)\right)=2 \eta_{2}\left(\mathrm{GL}_{n}\left(q_{0}\right)\right)$. 
Proof. Suppose first that $n=2^{t}, t>0$. By assumption 4 divides $q-1$, and by Lemma $3.2 \eta_{2}\left(\mathrm{GL}_{n}(q)\right)=2^{t}|q-1|_{2}$. If 4 divides $q_{0}-1$, then $\eta_{2}\left(\mathrm{GL}_{n}\left(q_{0}\right)\right)=$ $2^{t}\left|q_{0}-1\right|_{2}$, whereas if 4 divides $q_{0}+1$, then $\eta_{2}\left(\mathrm{GL}_{n}\left(q_{0}\right)\right)=2^{t}\left|q_{0}+1\right|_{2}$. As $\left|q_{0}^{2}-1\right|_{2}=$ $2\left|q_{0}-1\right|_{2}$ in the former case and $\left|q_{0}^{2}-1\right|_{2}=2\left|q_{0}+1\right|_{2}$ in the latter case, the result follows. If $2^{t+1}>n>2^{t}$, then $\eta_{2}\left(\mathrm{GL}_{n}(q)\right)=\eta_{2}\left(\mathrm{GL}_{2^{t}}(q)\right)=2 \eta_{2}\left(\mathrm{GL}_{2^{t}}\left(q_{0}\right)\right)=$ $2 \eta_{2}\left(\mathrm{GL}_{n}\left(q_{0}\right)\right)$, whence the statement.

Lemma 3.4. [35, Lemma 3.2] Let $p$ be an odd prime and $n>1$. Let $G=\mathrm{SL}_{n}(q)$, with $p \mid(q-1)$ or $G=\mathrm{SU}_{n}(q)$, with $p \mid(q+1)$. Then $G$ has no irreducible p-element.

Lemma 3.5. Let $q=q_{0}^{p^{k}}, k>0$, and $G=G(q) \in\left\{\mathrm{GL}_{n}(q), \mathrm{SL}_{n}(q), \mathrm{GU}_{n}(q)\right.$, $\left.\mathrm{SU}_{n}(q), \operatorname{Sp}_{2 n}(q), \Omega_{2 n+1}(q), \Omega_{2 n}^{ \pm}(q)\right\}$. Let $\Psi$ be the group of field automorphisms of $G$ of order $p^{k}, p>2$, and set $H=G \cdot \Psi$. Then the following holds:

(1) $\eta_{p}(H)=\max \left\{p^{(k-i)} \eta_{p}\left(G\left(q_{0}^{p^{i}}\right)\right): 0 \leq i \leq k\right\}$.

(2) If $p \mid q$, then $\eta_{p}(H)=p^{k} \cdot \eta_{p}(G)$.

(3) If $p \nmid q$, then $\eta_{p}(H)=\eta_{p}(G)$.

(4) If $p \nmid q$, then $\eta_{p}(L)=\eta_{p}(L \cdot \Psi)$, where $L$ is the simple non-abelian composition factor of $G$.

Proof. (1) The statement follows from [38, Corollary 14] and its immediate generalisation to any $G(q)$ as remarked in [31, taking into account the well known fact that, as $p$ is odd, $G\left(q_{0}\right) \subseteq G\left(q_{0}^{p}\right)$ (see [23, Table 4.5A]).

(2) The statement follows from (1) and the known fact that $\eta_{p}\left(G\left(q_{0}^{p^{i}}\right)\right)=\eta_{p}\left(G\left(q_{0}\right)\right)$ for $i=1, \ldots, k$. E.g., for an even stronger statement, [30, Lemma 2.32].

(3) The statement follows from [31, Lemma 3.10].

(4) It is well known that $L$ contains a subgroup isomorphic to a Sylow $p$-subgroup of $G$, unless $n$ is a $p$-power, $L=\operatorname{PSL}_{n}(q)$ and $p$ divides $(n, q-1)$ or $L=\operatorname{PSU}_{n}(q)$ and $p$ divides $(n, q+1)$. Let us consider the exceptional cases. By Lemma 3.4 every $p$-element of $\mathrm{SL}_{n}(q)$ and $\mathrm{SU}_{n}(q)$ is reducible. It follows that $\eta_{p}\left(\mathrm{SL}_{n}(q)\right) \leq$ $\eta_{p}\left(\mathrm{GL}_{n / p}(q)\right)$ and $\eta_{p}\left(\mathrm{SU}_{n}(q)\right) \leq \eta_{p}\left(\mathrm{GU}_{n / p}(q)\right)$. Moreover, there is an embedding $j: \mathrm{GL}_{n / p}(q) \rightarrow \mathrm{SL}_{n}(q)$ such that $j\left(\mathrm{GL}_{n / p}(q)\right.$ ) contains no scalar matrix (except the identity), which in turn yields an embedding $j: \mathrm{GL}_{n / p}(q) \rightarrow \mathrm{PSL}_{n}(q)$. Thus $\eta_{p}\left(\mathrm{PSL}_{n}(q)\right)=\eta_{p}\left(\mathrm{GL}_{n} / p(q)\right)=\eta_{p}\left(\mathrm{SL}_{n}(q)\right)$. Similarly, we have $\eta_{p}\left(\mathrm{PSU}_{n}(q)\right)=$ $\eta_{p}\left(\mathrm{GU}_{n / p}(q)\right)=\eta_{p}\left(\mathrm{SU}_{n}(q)\right)$. By $(3), \eta_{p}(L \cdot \Psi)=\eta_{p}\left(\mathrm{SL}_{n}(q) \cdot \Psi\right)=\eta_{p}\left(\mathrm{SL}_{n}(q)\right)=$ $\eta_{p}(L)$ for $L=\operatorname{PSL}_{n}(q)$, and similarly for $L=\operatorname{PSU}_{n}(q)$.

Lemma 3.6. Suppose $q=q_{0}^{2^{k}}$ where $k>0$ and $q$ is odd. Let $G=G(q) \in\left\{\operatorname{GL}_{n}(q)\right.$, $\left.\mathrm{SL}_{n}(q), \mathrm{PSL}_{n}(q)\right\}$ and let $\Psi$ be the group of field automorphisms of $G$ of order $2^{k}$. Set $H=G \cdot \Psi$. Then $\eta_{2}(H)=\eta_{2}(G)$.

Proof. By [38, Corollary 14], $\eta_{2}(H)=\max \left\{2^{(k-i)} \eta_{2}\left(G\left(q_{0}^{2^{i}}\right)\right): 0 \leq i \leq k\right\}$. For $G=$ $\mathrm{GL}_{n}(q)$, the statement then follows by iterated application of Corollary 3.3. Otherwise, the argument used to prove item (4) of Lemma 3.5 remains valid for $p=2$. (As $k>0$, Lemma 3.4 remains valid for $n=p=2$.) So $\eta_{2}\left(\operatorname{PSL}_{n}(q)\right)=\eta_{2}\left(\mathrm{GL}_{n / 2}(q)\right)=$ $\eta_{2}\left(\mathrm{SL}_{n}(q)\right)$. Again by Corollary 3.3 we get $\eta_{2}\left(\mathrm{GL}_{n / 2}(q)\right)=2^{k-i} \eta_{2}\left(\mathrm{GL}_{n / 2}\left(q_{0}^{2^{i}}\right)\right)$ for every $i$. It follows that $\eta_{2}(H)=\eta_{2}\left(\mathrm{GL}_{n / 2}(q)\right)=\eta_{2}(G)$, as claimed.

Lemma 3.7. Let $G=\mathrm{GL}_{n}(q), q$ odd, and suppose that $2^{t} \leq n<2^{t+1}$. Then $\eta_{2}(G)=2^{t}|q-1|_{2}$ if $4 \mid(q-1)$ and $2^{t}|q+1|_{2}$ if $4 \mid(q+1)$. 
Proof. By Lemma 3.2, $\eta_{2}(G)=\left|q^{e}-1\right|_{2} 2^{l}$, where $2^{l} e \leq n<2^{l+1} e$. If $e=1$, then $t=l$, and the statement follows. Let $e=2$. Then $\left|q^{2}-1\right|_{2}=|q-1|_{2}|q+1|_{2}=2|q+1|_{2}$ and $2^{l+1} \leq n<2^{l+2}$. So $l=t-1$, whence the statement.

\section{The GRoups $\operatorname{PSL}_{2}(q)$}

In this section we deal with the groups $L=\operatorname{PSL}_{2}(q)$. Set $G=\langle L, g\rangle$, where $1 \neq g \in$ Aut $L$ is a $p$-element, for some prime $p$. Note that here we may assume $g^{2} \neq 1$ in view of Lemma 2.10 and $g \in\left(G \backslash \mathrm{PGL}_{2}(q)\right)$, as the case $g \in \mathrm{PGL}_{2}(q)$ is settled in [6. Furthermore, recall that $L=\operatorname{PSL}_{2}(q)$ has no graph automorphism, and therefore Aut $L / \mathrm{PGL}_{2}(q) \cong \operatorname{Gal}\left(\mathbb{F}_{q} / \mathbb{F}_{r}\right)$. As above, let $\Phi=\operatorname{Gal}\left(\mathbb{F}_{q} / \mathbb{F}_{r}\right)$ and $p^{m}=|\Phi|_{p}$.

Theorem 4.1. Let $L=\mathrm{PSL}_{2}(q), q \geq 4$, and let $G=\langle L, g\rangle$, where $1 \neq g \in$ Aut $L$ is a p-element, for some prime $p$ and $g \notin \mathrm{PGL}_{2}(q)$. Let $\phi$ be a cross-characteristic irreducible projective $\mathbb{F}$-representation of $G$, which is non-trivial on L. Suppose that $g^{2} \neq 1$. Then $\phi(g)$ is not almost cyclic unless one of the following holds:

(1) $p=3, q=8,|g|=9$, and $\operatorname{dim} \phi=7,8$ if $\ell \neq 3$, whereas $\operatorname{dim} \phi=7$ if $\ell=3$.

(2) $p=2, q=4,|g|=4$ and either $\ell \neq 3,5$ and $\operatorname{dim} \phi=5$, or $\ell=3$ and $\operatorname{dim} \phi=4$, or $\ell=5$ and $\operatorname{dim} \phi=2,3,5$.

(3) $p=2, q=9$ and one of the following holds:

(i) If $\ell \neq 2,5$, then

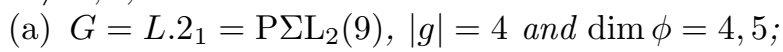

(b) $G=L .2_{2}=\mathrm{PGL}_{2}(9),|g|=8$ and $\operatorname{dim} \phi=6,8,9$;

(c) $G=L \cdot 2_{3}=M_{10},|g|=8$ and $\operatorname{dim} \phi=6,8,9$.

(ii) If $\ell=2$, then

(a) $G=L .2_{1}=\mathrm{P} \Sigma L_{2}(9),|g|=4$ and $\operatorname{dim} \phi=4$;

(b) $G=L \cdot 2_{2}=\mathrm{PGL}_{2}(9),|g|=8$ and $\operatorname{dim} \phi=8$;

(c) $G=L .2_{3}=M_{10},|g|=8$ and $\operatorname{dim} \phi=8$.

(iii) If $\ell=5$, then

(a) $G=L .2_{1}=\mathrm{P} \Sigma \mathrm{L}_{2}(9),|g|=4$ and $\operatorname{dim} \phi=4,5$;

(b) $G=L \cdot 2_{2}=\mathrm{PGL}_{2}(9),|g|=8$ and $\operatorname{dim} \phi=6,8$;

(c) $G=L .2_{3}=M_{10},|g|=8$ and $\operatorname{dim} \phi=8$.

Proof. Let $q=q_{0}^{p^{m}}$. By Lemma 2.8(1), $\operatorname{dim} \phi \geq \frac{q-1}{(q-1,2)}=\frac{q_{0}^{p^{m}}-1}{\left(q_{0}-1,2\right)}$, unless $q=4,9$. Note that if $g$ is semisimple, then $|g| \leq p^{m}|L|_{p}=p^{m}\left|\left(q_{0}^{2}\right)^{p^{m}}-1\right|_{p}=p^{m}\left|q_{0}^{2}-1\right|_{p}$ by Lemma $3.1(2)$.

Suppose that $p>2$ (and hence $q \neq 4,9$ ). If $g$ is unipotent, the result follows from [5, Lemma 4.13]. So, assume that $g$ is semisimple. Then, $|g| \leq p^{m}\left(q_{0}+1\right)$ and, by Lemma 2.3, $\alpha(g)=2$. If $\phi(g)$ is almost cyclic, the inequality $q_{0}^{p^{m}}-1 \leq$ $2\left(2, q_{0}-1\right)\left(p^{m}\left(q_{0}+1\right)-1\right)$ must hold by Lemma 2.4. However, this happens only for

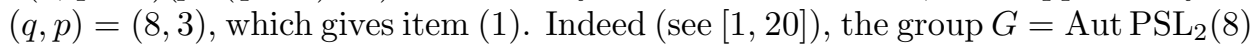
has irreducible representations of degree 7,8 over the complex numbers, in which the elements of order 9 are almost cyclic. This implies that the elements of order 9 are almost cyclic in every irreducible constituent of degree $>1$ of a Brauer reduction of any of these representations modulo any prime. If $\ell=3$, we only get constituents of degree 7 , whereas, if $\ell=7$ these are of degree 7 and 8 . Note that the case of $\ell=2$ is irrelevant here, but will appear for $G={ }^{2} G_{2}(3) \cong \mathrm{SL}_{2}(8) \cdot 3$.

Now, suppose that $p=2$ and consider first the case when $q>9$ is odd. By the above, $|g| \leq 2^{m+1}\left(q_{0}+1\right)$. Suppose that $\phi(g)$ is almost cyclic. Then $\frac{q-1}{2} \leq$ 
$\operatorname{dim} \phi \leq \alpha(g)(|g|-1)$ by Lemma 2.4. Thus, as $g^{2} \neq 1$, by Lemma 2.6 we must have $\frac{q_{0}^{2^{m}}-1}{2} \leq 2\left(2^{m+1}\left(q_{0}+1\right)-1\right)$. This inequality holds only if either $m=1$ and $q_{0}=3,5,7,9,11,13$, or $m=2$ and $q_{0}=3$. However, $\eta_{2}\left(\mathrm{PSL}_{2}(81)\right)=16$, contradicting Lemma 2.4. Similarly, we can rule out $q=121$ and $q=169$, since $\eta_{2}\left(\mathrm{PSL}_{2}(121)\right) \leq 16$ and $\eta_{2}\left(\mathrm{PSL}_{2}(169)\right) \leq 16$. So, we are left with the cases $q=25,49$.

Next, suppose that $q>4$ is even. Then $\operatorname{dim} \phi \geq q-1$ and clearly $|g| \leq 2^{m+1}$. If $\phi(g)$ is almost cyclic, then $q-1 \leq \operatorname{dim} \phi \leq \alpha(g)(|g|-1)$. By Lemma 2.3 (1), $\alpha(g) \leq 4$. Thus, since $g^{2} \neq 1, q_{0}^{2^{m}}-1 \leq 4\left(2^{m+1}-1\right)$, which holds if and only if $q_{0}=2$ and $m=1,2$, i.e. if and only if $q=4,16$.

In conclusion, we are left with the cases where $q \in\{4,9,16,25,49\}$.

1) Suppose $q=4$. Then $|g|=4, \alpha(g)=2$, and using the packages GAP and MAGMA we get item (2) of the statement.

2) Suppose $q=9$. Then $|g|=4,8$ and $\alpha(g)=2$. If $\ell \neq 2$, using the packages GAP and MAGMA we get the results recorded in the statement, item (3). So, suppose $\ell=2$. Assume first $|g|=4$. Then $\phi(g)$ almost cyclic implies $\operatorname{dim} \phi \leq 6$,

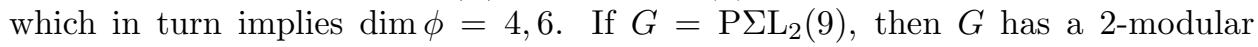
representation of degree 4 in which $\phi(g)$ is actually cyclic, whereas 3.G has a faithful 2 -modular representation of degree 6 in which $\phi(g)$ is almost cyclic. There are no other occurrences for $|g|=4$. Next, assume that $|g|=8$. Then $\phi(g)$ almost cyclic implies $\operatorname{dim} \phi \leq 14$. Then either $G=\mathrm{PGL}_{2}(9)$ or $G=M_{10}$. In the first case there is a 2-modular representation of $G$ of degree 8 in which $\phi(g)$ is almost cyclic, and a faithful 2-modular representation of 3.G of degree 6 in which $\phi(g)$ is almost cyclic. In the latter case, there is a 2-modular representation of $G$ of degree 8 in which $\phi(g)$ is almost cyclic, and two faithful 2-modular representations of 3.G of degree 6 and 9 respectively, in which $\phi(g)$ is almost cyclic.

3) Suppose $q=16$. Then $|g|=4,8$ and $\alpha(g)=2$. Since $\operatorname{dim} \phi \geq 15, \phi(g)$ cannot be almost cyclic by Lemma 2.4. So this case is ruled out.

4) Suppose $q=25$. Then $|g|=4,8$ and $\alpha(g)=2$. Since $\operatorname{dim} \phi \geq 12$, by Lemma

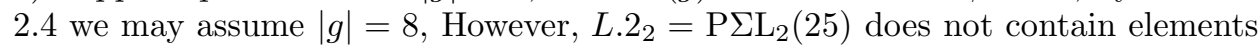
of order 8 . So, we are left to consider the projective representations of $L .2_{1}=$ $\mathrm{PGL}_{2}(25)$ and of $L .2_{3}$. Making use of GAP we see that if $\ell \neq 2$, then $\phi(g)$ is not almost cyclic. On the other hand, if $\ell=2$, then $\operatorname{dim} \phi \geq 24$, and hence $\phi(g)$ is not almost cyclic. So this case is ruled out.

5) Suppose $q=49$. Then $|g|=4,8,16$ and $\alpha(g)=2$. Since $\operatorname{dim} \phi \geq 24$, again by Lemma 2.4 we may assume $|g|=16$. However, $L .2_{2}=\mathrm{P} \Sigma \mathrm{L}_{2}(49)$ does not contain elements of order 16 . So, we are left to consider the projective representations of $L .2_{1}=\mathrm{PGL}_{2}(49)$ and of $L .2_{3}$. Making use of GAP we see that if $\ell \neq 2,3 \phi(g)$ is not almost cyclic. On the other hand, if $\ell=2$, then $\operatorname{dim} \phi \geq 48$, and hence $\phi(g)$ is not almost cyclic. So, also this last case is ruled out.

5. The GRoups $\operatorname{PSL}_{n}(q), n>2 ; \operatorname{PSU}_{n}(q), n>2 ; \operatorname{PSp}_{2 n}(q), n>1, q$ ODD

In this section we consider the simple groups $L=\operatorname{PSL}_{n}(q), n>2, \operatorname{PSU}_{n}(q)$, $n>2$ with $(n, q) \neq(3,2), \operatorname{PSp}_{2 n}(q), q$ odd.

Let $1 \neq g \in$ Aut $L$ be a $p$-element, set $G=\langle L, g\rangle$ and let $\phi$ be a crosscharacteristic projective irreducible representation of $G$ that is non-trivial on $L$. Observe that if the restriction of $\phi$ to $L$ has a constituent which is not a Weil representation of $L$, a lower bound of $\operatorname{dim} \phi$ is provided by Lemma 2.9. On the other 
hand, the occurrence of almost cyclic elements in the Weil representations of the groups $L$ listed above is thoroughly dealt with in our earlier paper [6]. There, it is also shown that they extend to the subgroup $A_{d}$ of Aut $L$ generated by all inner and diagonal automorphisms. Now, suppose that $g \in A_{d} \backslash L$ and $\phi$ restricted to $L$ decomposes into the sum of, say, $t$ constituents which are all Weil representations of $L$. By Clifford's theorem these constituents are all $g$-conjugates. However, they extend to $A_{d}$. Hence $t=1$; in other words, $\phi$ itself is a Weil representation of $A_{d}$, and is disposed of in [6. In view of this, this section will be mainly concerned with answering the following cases: (A) the case when the restriction of $\phi$ to $L$ has a constituent which is not a Weil representation of $L$; (B) the case when $g \notin A_{d}$ and all the constituents of the restriction of $\phi$ to $L$ are Weil representations of $L$.

5.1. Case (A). Apart from very few groups, case (A) is answered by the following.

Lemma 5.1. Let $L$ be one of the following simple groups:

(1) $\operatorname{PSL}_{n}(q)$, where $n \geq 3$ and $(n, q) \notin\{(3,2),(3,4),(4,2)\}$;

(2) $\operatorname{PSU}_{n}(q)$, where $n \geq 3$ and $(n, q) \notin\{(3,3),(3,4),(4,2),(4,3),(5,2)\}$;

(3) $\operatorname{PSp}_{2 n}(q)$, where $n \geq 2$, $q$ is odd and $(n, q) \neq(2,3)$.

Let $1 \neq g \in$ Aut $L$ be a p-element for some prime $p$ and set $G=\langle L, g\rangle$. Let $\phi$ be a cross-characteristic projective irreducible representation of $G$ that is non-trivial on $L$. If the restriction of $\phi$ to $L$ has a constituent which is not a Weil representation of $L$, then $\phi(g)$ is not almost cyclic.

Proof. Suppose that $\phi(g)$ is almost cyclic. By Lemma 2.4 the following bound must be met:

$$
\operatorname{dim} \phi \leq \alpha(g) \cdot(|g|-1)
$$

Since the restriction of $\phi$ to $L$ has a constituent which is not a Weil representation of $L$, lower bounds for $\operatorname{dim} \phi$ are provided by Lemma 2.9. Upper bounds for $\alpha(g)$ are given in Propositions 2.2 and 2.3 . Finally, by [12, Theorem 2.16] we have $|g| \leq \mu(L)$, where

$$
\mu(L)=\left\{\begin{array}{cl}
\frac{q^{n}-1}{q-1} & \text { if } L=\operatorname{PSL}_{n}(q), \\
q^{n-1}-1 & \text { if } L=\operatorname{PSU}_{n}(q) \text { with } n \text { odd and } q \text { not prime, } \\
q^{n-1}+q & \text { if } L=\operatorname{PSU}_{n}(q) \text { with } n \text { odd and } q \text { prime, } \\
q^{n-1}+1 & \text { if } L=\operatorname{PSU}_{n}(q) \text { with } n \text { even and } q>2, \\
4\left(2^{n-3}+1\right) & \text { if } L=\operatorname{PSU}_{n}(q) \text { with } n \text { even and } q=2, \\
\frac{q^{n+1}}{q-1} & \text { if } L=\operatorname{PSp}_{2 n}(q) .
\end{array}\right.
$$

Suppose first that $L=\operatorname{PSL}_{n}(q)$. If $n=3$ and $q \geq 5$, then $\alpha(g) \leq 4$. The bound $\vartheta_{3}^{+}(q) \leq 4\left(q^{2}+q\right)$ only holds for $q=5,7,13$. Next, assume that $n=4$ and $q \geq 4$. In this case, $\alpha(g) \leq 6$ : the bound $\vartheta_{4}^{+}(q) \leq 6\left(q^{3}+q^{2}+q\right)$ only holds for $q=4,5,7,9,11,13$. Now, assume that $n \geq 5$ and $(n, q) \neq(6,2),(6,3)$. In this case, the inequality $\vartheta_{n}^{+}(q) \leq n \frac{q^{n}-q}{q-1}$ only holds for $(n, q)=(5,2)$.

If $L=\mathrm{PSL}_{3}(3)$, then $|g| \in\{2,3,4,8,13\}$ and $\operatorname{dim} \phi \geq 16$. If $|g| \leq 3$, then $\alpha(g) \leq 3$, whereas if $|g| \geq 4$, then $\alpha(g)=2$. Thus we readily get a contradiction for $|g| \neq 13$. Likewise, for $|g|=13$, using the GAP package we see that $\phi(g)$ is not almost cyclic.

If $L \in\left\{\mathrm{PSL}_{4}(3), \mathrm{PSL}_{5}(2), \mathrm{PSL}_{6}(2), \mathrm{PSL}_{6}(3)\right\}$ we use the following data to get a contradiction with the bound (5.1): 
(i) $L=\mathrm{PSL}_{4}(3):|g| \in\{2,3,4,5,8,9,13\}$ and $\operatorname{dim} \phi \geq 26$. If $|g| \geq 5$, then $\alpha(g)=2 ;$ if $|g| \leq 4$ then $\alpha(g) \leq 6$.

(ii) $L=\mathrm{PSL}_{5}(2):|g| \in\{2,3,4,5,7,8,16,31\}$ and $\operatorname{dim} \phi \geq 75$. If $|g| \geq 5$, then $\alpha(g)=2 ;$ if $|g| \leq 4$ then $\alpha(g) \leq 5$.

(iii) $L=\mathrm{PSL}_{6}(2):|g| \in\{2,3,4,5,7,8,9,16,31\}$ and $\operatorname{dim} \phi \geq 61$. If $|g| \geq 5$, then $\alpha(g)=2$, whereas if $|g| \leq 4$, then $\alpha(g) \leq 6$.

(iv) $L=\mathrm{PSL}_{6}(3):|g| \in\{2,3,4,5,7,8,9,11,13,16,121\}$ and $\operatorname{dim} \phi \geq 362$. If $|g| \geq 9$ then $\alpha(g)=2$; if $|g| \leq 8$ then $\alpha(g) \leq 6$.

Next, suppose that $L=\operatorname{PSU}_{n}(q)$. If $n=3$ and $q \geq 5$, the bound $\vartheta_{3}^{-}(q) \leq$ $3\left(\mu\left(\operatorname{PSU}_{3}(q)\right)-1\right)$ only holds for $q=5,8,11,17$. If $n=4$ and $q \geq 4$, the bound $\vartheta_{4}^{-}(q) \leq 6\left(\mu\left(\mathrm{PSU}_{4}(q)\right)-1\right)$ only allows the following possibilities: $q=4,5,7,9,11$. If $n \geq 5$ and $(n, q) \neq(6,2)$, the bound $\vartheta_{n}^{-}(q) \leq n\left(\mu\left(\mathrm{PSU}_{n}(q)\right)-1\right)$ only holds when $(n, q) \in\{(5,2),(5,3),(7,2)\}$.

If $L=\operatorname{PSU}_{6}(2)$, then $|g| \in\{2,3,4,5,7,8,9,11,16\}$ and $\operatorname{dim} \phi \geq 21$. If $|g| \geq 5$, then $\alpha(g)=2$, while if $|g| \leq 4$ then $\alpha(g) \leq 6$. Thus, if $|g| \neq 16$, by Lemma $2.4 \phi(g)$ is not almost cyclic. If $|g|=16(g \notin L)$, we need to examine the representations $\phi$ such that $21 \leq \operatorname{dim} \phi \leq 30$. Using the GAP package, we find that $\phi(g)$ is never almost cyclic.

Finally, suppose that $L=\operatorname{PSp}_{2 n}(q)$. If $n=2$ and $q \geq 5$, then the bound $\sigma_{2}(q) \leq$ $5 \frac{q^{3}-q+1}{q-1}$ only holds for $q=5,7,9,11$. If $n \geq 3$, the bound $\sigma_{n}(q) \leq 2 n \frac{q^{n+1}-q+1}{q-1}$ only holds for $(n, q) \in\{(3,3),(4,3)\}$.

If $L=\mathrm{PSp}_{4}(5)$, then $|g| \in\{2,3,4,5,8,13\}$ and $\operatorname{dim} \phi \geq 40$. If $|g|=8,13$, then $\alpha(g)=2$, whereas if $|g| \leq 5$, then $\alpha(g) \leq 5$. In both cases Lemma 2.4 yields a contradiction.

In view of the above, we are left to examine the groups $L$ listed in Table 1 below. But again, in each case, in view of the data shown in table, we conclude by Lemma 2.4 that $\phi(g)$ cannot be almost cyclic.

\begin{tabular}{c|c|c|c||c|c|c|c}
$L$ & $|g| \leq$ & $\alpha(g) \leq$ & $\operatorname{dim} \phi \geq$ & $L$ & $|g| \leq$ & $\alpha(g) \leq$ & $\operatorname{dim} \phi \geq$ \\
\hline $\mathrm{PSL}_{3}(5)$ & 31 & 3 & 96 & $\mathrm{PSL}_{3}(7)$ & 19 & 4 & 96 \\
$\mathrm{PSL}_{3}(13)$ & 61 & 4 & 672 & $\mathrm{PSL}_{4}(4)$ & 17 & 6 & 189 \\
$\mathrm{PSL}_{4}(5)$ & 31 & 6 & 248 & $\mathrm{PSL}_{4}(7)$ & 25 & 6 & 1026 \\
$\mathrm{PSL}_{4}(9)$ & 41 & 6 & 2912 & $\mathrm{PSL}_{4}(11)$ & 61 & 6 & 6650 \\
$\mathrm{PSL}_{4}(13)$ & 61 & 6 & 13176 & $\mathrm{PSU}_{3}(5)$ & 8 & 3 & 28 \\
$\mathrm{PSU}_{3}(8)$ & 19 & 3 & 105 & $\mathrm{PSU}_{3}(11)$ & 37 & 3 & 260 \\
$\mathrm{PSU}_{3}(17)$ & 32 & 3 & 912 & $\mathrm{PSU}_{4}(4)$ & 17 & 6 & 220 \\
$\mathrm{PSU}_{4}(5)$ & 13 & 6 & 272 & $\mathrm{PSU}_{4}(7)$ & 43 & 6 & 1074 \\
$\mathrm{PSU}_{4}(9)$ & 64 & 6 & 2992 & $\mathrm{PSU}_{4}(11)$ & 64 & 6 & 6770 \\
$\mathrm{PSU}_{5}(3)$ & 61 & 5 & 324 & $\mathrm{PSU}_{7}(2)$ & 43 & 7 & 320 \\
$\mathrm{PSp}_{4}(7)$ & 25 & 5 & 126 & $\mathrm{PSp}_{4}(9)$ & 41 & 5 & 288 \\
$\mathrm{PSp}_{4}(11)$ & 61 & 5 & 550 & $\mathrm{PSp}_{6}(3)$ & 13 & 6 & 78 \\
$\mathrm{PSp}_{8}(3)$ & 41 & 8 & 780 & & & &
\end{tabular}

TABLE 1. Bounds for $|g|, \alpha(g)$ and $\operatorname{dim} \phi$ for some simple groups. 
5.2. Case (B). We now consider case (B): $g \notin A_{d}$ and all the constituents of the restriction of $\phi$ to $L$ are Weil representations of $L$. We start with recording some arithmetical inequalities to be used when applying Lemma 2.4

Lemma 5.2. Let $r$ be a prime, $q=r^{a}$.

(1) Let $n \geq 5$. Then $\frac{q^{n}-1}{q-1}-2>n(q-1)$. Furthermore, $\frac{q^{3}-1}{q-1}-2>4(q-1)$ and $\frac{q^{4}-1}{q-1}-2>7(q-1)$.

(2) Let $n \geq 3$ and $q$ odd. Then $\frac{q^{n}-1}{2}>2 n(q-1)$. Furthermore, $\frac{q^{2}-1}{2}>6(q-1)$ unless $3 \leq q \leq 11$.

(3) Let $n \geq 5$ be odd. Then $\frac{q^{n}-q}{q+1}>n(q-1)$. Furthermore, $\frac{q^{3}-q}{q+1}>4(q-1)$, unless $q=2,3,4$.

(4) Let $n \geq 6$ be even. Then $\frac{q^{n}-1}{q+1}>n(q-1)$. Furthermore, $\frac{q^{4}-1}{q+1}>6(q-1)$, unless $q=2$.

Proof. Elementary straightforward computations.

Lemma 5.3. Let $L$ be one of the simple groups $\operatorname{PSL}_{n}(q), n>2 ; \operatorname{PSU}_{n}(q), n>2$; $\operatorname{PSp}_{2 n}(q), n>1, q$ odd. Let $G=\langle g, L\rangle \subseteq$ Aut $L$, where $g$ is a p-element. Let $\phi$ be a cross-characteristic irreducible projective $\mathbb{F}$-representation of $G$ non-trivial on $L$, and suppose that $p$ is coprime to $|L|$. Then $\phi(g)$ is not almost cyclic.

Proof. Observe that if $p$ is coprime to $|L|$, then $p>3$, and hence $|g|$ divides $|\Phi|$. This implies $3<|g|<q-1$. Now, by Lemma 2.8 the minimum degree of a non-trivial irreducible projective representation of $L$ is not less than the number at the left hand side of the items (1) to (4) of Lemma 5.2 unless $(n, q) \in\{(3,2),(3,4),(4,2),(4,3)\}$ in case (1), and $(n, q) \in\{(4,2),(4,3)\}$ in case (4). However, all these exceptions occur for $q=r$, that is $|\Phi|=1$, or $q=4$, and therefore are irrelevant here. Similarly, all the exceptions listed in cases (2), (3) and (4) of Lemma 5.2 are irrelevant here. Furthermore, by Lemmas 2.2 and 2.3. $\alpha(g) \leq n$ if $L$ is not symplectic, and $\alpha(g) \leq 2 n$ in the symplectic case, with some exceptions described there. Again, as $2<|g|<$ $q-1$, all those exceptions are ruled out. By Lemma 5.2 and Lemma 2.4, it follows that $\phi(g)$ is not almost cyclic.

We now suppose that $p$ is a prime dividing $|L|$ and distinguish two cases depending on the divisibility of $q$ by $p$.

5.2.1. Case $(p, q)=1$.

Lemma 5.4. Let $L=\operatorname{PSL}_{n}(q)$, with $n>2$, and let $p>2$ be such that $p$ divides $|L|$ and $(p, q)=1$. Let $1 \neq g \in$ Aut $L$ be a p-element and let $G=\langle g, L\rangle$. Suppose that $g \notin A_{d}$. Then $n|g|<\frac{q^{n}-1}{q-1}-2$, and $g$ is not almost cyclic in any cross-characteristic projective irreducible representation of $G$ that is non-trivial on $L$.

Proof. Let $H=\mathrm{GL}_{n}(q)$. As $p>2, g \in(H \cdot \Phi) / Z(H) \subseteq$ Aut $L$. By Lemmas 3.2 and 3.5. $|g| \leq \eta_{p}(H)=p^{t}\left|q^{e}-1\right|_{p} \leq \frac{n}{e}\left(q^{e}-1\right)$, where $p^{t} e \leq n<p^{t+1} e$. Observe that, since $p$ is odd, the assumption $g \notin A_{d}$ implies that $|\Phi|_{p}=p^{m}>1$. This in turn implies $q \geq 8$, and hence $\frac{q^{n}-1}{q-1}-2$ is the minimum degree of a non-trivial crosscharacteristic projective irreducible representation of $L$ (see Lemma 2.8). Notice that if $e<\frac{n}{2}$, then $\frac{n^{2}}{e}\left(q^{e}-1\right)<\frac{q^{n}-1}{q-1}-2$. Therefore the statement is proven for $n>2 e$. 
Next, suppose that $n \leq 2 e$, and observe that the assumption $n>2$ implies $e>1$. Since $p^{t} e \leq n \leq 2 e$ and $p>2$, it follows that $p^{t}=1$. Let $q_{1}$ be such that $q_{1}^{p^{m}}=q^{e}$. By Lemma 3.1] we get $\left|q^{e}-1\right|_{p}=p^{m}\left|q_{1}-1\right|_{p}$, whence $|g| \leq \eta_{p}(H) \leq p^{m}\left(q_{1}-1\right)$. We need to show that $n p^{m}\left(q_{1}-1\right)<\frac{q^{n}-1}{q-1}-2$, and for this it is enough to show that $n p^{m}\left(q_{1}-1\right)<q^{n-1}+q^{n-2}-2$. Since $e \leq n$ and $e<p$ (note that $p$ is a Zsigmondy prime for the pair $(q, e))$, it suffices to show that $2 p^{m+1}\left(q_{1}-1\right)<$ $q^{e-1}+q^{e-2}-2=q_{1}^{p^{m}-1}+q_{1}^{p^{m}-2}-2$. This is true except when $m=1$ and either $p=3$ or $\left(p, q_{1}\right) \in\{(5,2),(7,2)\}$. If $p=5,7$ and $q_{1}=2$, then $q^{e}=2^{5}, 2^{7}$, whence $q=2$ and $e=p$, a contradiction, as $e<p$. If $p=3$, then $2 \leq e<p$ yields $e=2$. Thus, we are left to consider the cases $q^{2}=q_{1}^{3}$ and $n=3,4$. Notice that $12\left(q_{1}-1\right)<q_{1}^{3}+5$. It follows that, for $n=3$, the inequality $n p^{m}\left(q_{1}-1\right)<\frac{q^{n}-1}{q-1}-2$ reduces to $9\left(q_{1}-1\right)<q^{2}+q-1$, and in fact $9\left(q_{1}-1\right)<12\left(q_{1}-1\right)<q_{1}^{3}+5 \leq q^{2}+q-1$, as $q \geq 8$. For $n=4$, the same inequality reduces to $12\left(q_{1}-1\right)<q^{3}+q^{2}+q-1$. Which is true, since $12\left(q_{1}-1\right)<q_{1}^{3}+5<q^{3}+q^{2}+q-1$. This ends the proof.

Lemma 5.5. Let $L=\operatorname{PSL}_{n}(q)$ with $n>2, q$ odd. Let $g \in$ Aut $L$ be a 2-element such that $g^{2} \neq 1$, and let $G=\langle g, L\rangle$. Then $g$ is not almost cyclic in any crosscharacteristic projective irreducible representation of $G$ that is non-trivial on $L$.

Proof. Let $H=\mathrm{GL}_{n}(q)$, and let $\Gamma$ be the group generated by the inverse-transpose automorphism of $H$. Clearly $|\Gamma|=2$. Then $(H \cdot \Phi \cdot \Gamma) / Z(H) \cong$ Aut $L$, and therefore it suffices to prove the statement for $H \cdot \Phi \cdot \Gamma$ in place of Aut $L$. Obviously, $\eta_{2}(H \cdot \Phi \cdot \Gamma) \leq 2 \eta_{2}(H \cdot \Phi)$. By Lemma 3.6, $\eta_{2}(H \cdot \Phi)=\eta_{2}(H)$. Let $m \geq 0$ such that $q=q_{0}^{2^{m}}$, where $q_{0}$ is not a square. By Corollary 3.3 and Lemma 3.7 we get $\eta_{2}(H)=2^{m} \eta_{2}\left(\mathrm{GL}_{n}\left(q_{0}\right)\right) \leq 2^{m+t}\left(q_{0}+1\right)$, where $t$ is such that $2^{t} \leq n<2^{t+1}$ (notice that $t \geq 1$ as $n>2)$. Hence, we have $\eta_{2}(H \cdot \Phi \cdot \Gamma) \leq 2^{m+t+1}\left(q_{0}+1\right)$.

We now show that if $n \geq 5$ and $(n, q) \neq(5,3)$, then $n \cdot 2^{m+t+1}\left(q_{0}+1\right)<\frac{q^{n}-1}{q-1}-$ 2. Under these restrictions, the statement of the lemma follows, by Proposition 2.2 and Lemmas 2.4 and 2.8. Clearly, $n \cdot 2^{m+t+1} \leq 2^{m+2 t+2}$ and $\frac{q^{n}-1}{q-1}-2 \geq$ $q^{n-1}+q^{n-2}-1 \geq q^{2^{t}-1}+q^{2^{t}-2}-1$, so it suffices to prove that $2^{m+2 t+2}\left(q_{0}+1\right)<$ $q_{0}^{2^{m+t}-2^{m}}+q_{0}^{2^{m+t}-2^{m+1}}-1$. Direct computation shows that this is true except when $(m, t)=(0,2)$ and $q_{0} \leq 7$. So, suppose that $m=0$ and $t=2$ : we have to prove that $8 n\left(q_{0}+1\right)<\frac{q_{0}^{n}-1}{q_{0}-1}-2$ for $n=5,6,7$. This is obviously true except when $\left(n, q_{0}\right)=(5,3)$.

Now, suppose that $n=3$. In this case the statement will be proven if we show that $2^{m+4}\left(q_{0}+1\right)<q_{0}^{2^{m+1}}+q_{0}^{2^{m}}-1$. Computation shows that this is true except when either $m=0$ and $q_{0} \leq 13$ or $\left(m, q_{0}\right)=(1,3)$.

Next, suppose that $n=4$. For $q \neq 3$, the statement will be proven once we show that

$$
6 \cdot 2^{m+3}\left(q_{0}+1\right)<q_{0}^{3 \cdot 2^{m}}+q_{0}^{2^{m+1}}+q_{0}^{2^{m}}-1
$$

Now, if $m \geq 3$, then $6 \cdot 2^{m+3}<3^{3 \cdot 2^{m}-1}$ and $6 \cdot 2^{m+3}<3^{2^{m+1}}$. Direct computation for $m \leq 2$ shows that (5.2) holds except for $m=0$ and $q_{0}=3,5$.

We finally deal with the exceptions arisen above. Let $\phi$ be a cross-characteristic projective irreducible representation of $G$ non-trivial on $L$. In the cases listed in the following table we easily get a contradiction by Lemma 2.4. 


\begin{tabular}{c|c|c||c|c|c}
$L$ & $\eta_{2}(L)$ & $\operatorname{dim} \phi \geq$ & $L$ & $\eta_{2}(L)$ & $\operatorname{dim} \phi \geq$ \\
\hline $\mathrm{PSL}_{3}(5)$ & 8 & 29 & $\mathrm{PSL}_{3}(9)$ & 16 & 89 \\
$\mathrm{PSL}_{3}(11)$ & 8 & 131 & $\operatorname{PSL}_{3}(13)$ & 8 & 181 \\
$\mathrm{PSL}_{4}(5)$ & 8 & 154 & $\operatorname{PSL}_{5}(3)$ & 16 & 119
\end{tabular}

Thus, we are left with the cases where $L=\mathrm{PSL}_{3}(3), \mathrm{PSL}_{3}(7)$ and $\mathrm{PSL}_{4}(3)$.

If $L=\mathrm{PSL}_{3}(3)$, then $\eta_{2}(L)=8$ and $\operatorname{dim} \phi \geq 11$. Using GAP we see that if $|g|=4$, then $\alpha(g)=2$. By Lemma 2.4, we conclude that $\phi(g)$ is not almost cyclic. If $|g|=8$, we are left to consider the case $11 \leq \operatorname{dim} \phi \leq 14$. Again using GAP, we see that $\phi(g)$ is not almost cyclic.

If $L=\mathrm{PSL}_{3}(7)$, then $\eta_{2}(L)=16$ and $\operatorname{dim} \phi \geq 55$. Using GAP, we see that for any 2-element $g$ such that $g^{2} \neq 1$ we have $\alpha(g)=2$, and hence again, by Lemma 2.4. $\phi(g)$ is not almost cyclic.

Finally, if $L=\mathrm{PSL}_{4}(3)$, then $\eta_{2}(L)=8$ and $\operatorname{dim} \phi \geq 26$. If $|g|=8$, then $\alpha(g)=2$; if $|g|=4$, then $\alpha(g) \leq 3$. In both cases, by Lemma $2.4 \phi(g)$ is not almost cyclic. This ends the proof.

Let $L=\operatorname{PSU}_{n}(q), n>2$, and let $p$ be a prime such that $(p, q)=1$ and $p$ divides $|L|$. Let $\Phi=\operatorname{Gal}\left(\mathbb{F}_{q} / \mathbb{F}_{r}\right)$, and set $p^{m}=|\Phi|_{p}$. Thus $p^{m}<r^{p^{m}} \leq q$. Let $g \in$ Aut $L$ be a $p$-element and set $G=\langle L, g\rangle$. Recall that, by Propositions 2.2 and 2.3. $\langle L, g\rangle$ is generated by $n$ conjugates of $g$. Finally, set $\Phi_{2}=\operatorname{Gal}\left(\mathbb{F}_{q^{2}} / \mathbb{F}_{r}\right)$, so that Aut $L=A_{d} \cdot \Phi_{2}$.

Lemma 5.6. Let $p>2, m>0$ and $e \neq 2(\bmod 4)$. Let $1 \neq g \in$ Aut $L$ be a p-element. Set $G=\langle L, g\rangle$. Then $n|g|<\left(q^{n}-q\right) /(q+1)$ if $n$ is odd, $n|g|<$ $\left(q^{n}-1\right) /(q+1)$ if $n$ is even, and $\phi(g)$ is not almost cyclic in any cross-characteristic irreducible projective representation $\phi$ of $G$ non-trivial on $L$.

Proof. Let $H=\mathrm{GU}_{n}(q)$. As $e=e_{p}(q) \neq 2, p$ is coprime to $q+1=|Z(H)|$. It follows that $\left|A_{d} / L\right|$ is coprime to $p$. So $\left(H \cdot \Phi_{2}\right) / Z(H) \subseteq$ Aut $L$ contains a Sylow $p$-subgroup of Aut $L$.

It is known (see for instance [17, Lemma 3.5]), that $H$ contains a subgroup isomorphic to $H_{1}:=\mathrm{GL}_{\lfloor n / 2\rfloor}\left(q^{2}\right)$, where $\lfloor n / 2\rfloor$ is the integral part of $n / 2$, and $\left|H: \mathrm{GL}_{\lfloor n / 2\rfloor}(q)\right|$ is coprime to $p$. Moreover, $H_{1}$ can be chosen to be invariant under $\Phi_{2}$, and therefore $H_{1} \cdot \Phi_{2}$ contains a Sylow $p$-subgroup of $H \cdot \Phi_{2}$. So, by Lemma 3.5. $|g| \leq \eta_{p}\left(H_{1}\right)$. Set $l=\lfloor n / 2\rfloor$ (that is, $l=n / 2$ if $n$ is even, $l=(n-1) / 2$ if $n$ is odd). As $p>2$, we have $|\Phi|_{p}=\left|\Phi_{2}\right|_{p}>1$; in particular, $q \geq r^{p} \geq 8$.

Note that $\left|q^{2 e}-1\right|_{p}=\left|q^{e}-1\right|_{p}$ if $e$ is odd, and $e_{p}\left(q^{2}\right)=e_{p}(q) / 2$ if $e$ is even. So in both cases $\left|\left(q^{2}\right)^{e_{p}\left(q^{2}\right)}-1\right|_{p}=\left|q^{e}-1\right|_{p}$. Therefore, by Lemma 3.2. $|g| \leq$ $\lfloor n / 2\rfloor \cdot\left|q^{e}-1\right|_{p}$, whence $|g| \leq\lfloor n / 2\rfloor \cdot\left|q^{e}-1\right|_{p} \leq l\left(q^{l}-1\right)$ (since $\left.e \leq l\right)$.

Suppose first that $n$ is even. In order to prove the statement, by Propositions 2.2 and 2.3. Lemma 2.4 and Lemma 2.8, it will be enough to show that $n|g|<$ $\left(q^{n}-1\right) /(q+1)$. For this purpose, it suffices to show that $(2 l) \cdot l \cdot(q+1)<q^{l}+1$. This is true if $l>2$, unless $l=3, q \leq 4 ; l=4, q \leq 3 ; 5 \leq l \leq 8, q=2$. However, all these exceptions are ruled out, since $q \geq r^{p}$. Next, suppose that $l=2$, so that $n=4$ and $e=1$, that is, $p \mid(q-1)$. Then $8(q+1)<q^{2}+1$, unless $q \leq 8$. As $q \geq r^{p}$, this forces $q=8$. However, as $p \mid(q-1)$, this in turn implies $p=7$, a contradiction.

Now, suppose that $n$ is odd. In this case, it will be enough to show that $n|g|<$ $\left(q^{n}-q\right) /(q+1)$. For this purpose, it suffices to show that $(2 l+1) \cdot l \cdot(q+1)<q^{l}+1$ 
(since $q^{n-1}<q^{n}-q$ ). This is true if $l>2$, unless $l=3, q \leq 5 ; l=4, q \leq 3$; $5 \leq l \leq 9, q=2$. However, all these exceptions are ruled out, since $q \geq r^{p}$. If $l=2$, that is, $n=5$, we have $10\left(q^{2}-1\right)<\left(q^{5}-q\right) /(q+1)$, unless $q \leq 3$. If $\bar{l}=1$, that is, $n=3,|g| \leq(q-1)$ and the inequality $3(q-1)<q(q-1)$ holds unless $q \leq 3$. As $q \geq 8$, we are done.

Lemma 5.7. Let $L=\operatorname{PSU}_{n}(q)$, with $n>2$. Let $1 \neq g \in$ Aut $L$ be a p-element, for some prime $p>2$ such that $e_{p}(q) \equiv 2(\bmod 4)$, and let $G=\langle g, L\rangle$. Then $g$ is not almost cyclic in any cross-characteristic projective irreducible representation of $G$ that is non-trivial on $L$, unless $n>3$ and $|\Phi|_{p}=1$ (and hence $g \in A_{d}$ ).

Proof. First of all, we observe that, by Lemmas 3.2 and $3.5|g| \leq \eta_{p}\left(\mathrm{GL}_{n}\left(q^{2}\right) \cdot \Phi_{2}\right)=$ $\eta_{p}\left(\mathrm{GL}_{n}\left(q^{2}\right)\right)=p^{t}\left|\left(q^{2}\right)^{e^{\prime}}-1\right|_{p}$, where $e^{\prime}=e_{p}\left(q^{2}\right)$ and $p^{t} e^{\prime} \leq n<p^{t+1} e^{\prime}$. By the definition of $e, e=2 e^{\prime}$ and, since $e$ is even, we obtain that $|g| \leq p^{t}\left|q^{e}-1\right|_{p}=$ $p^{t}\left|q^{e / 2}+1\right|_{p}$.

Assume first that $n=3$. Then $e_{p}(q)=2$, that is, $p$ divides $q+1$. By Lemma 2.8 (3), $\operatorname{dim} \phi \geq q^{2}-q$. Thus, the statement will be proven once we show that $3|g| \leq q^{2}-q$ (see Proposition 2.3(3)). Since $p \mid(q+1)$, we have $e=2$, whence $|g| \leq p^{t}\left|q^{2}-1\right|_{p}=p^{t}|q+1|_{p} \leq p^{t}(q+1)$. If $p>3$, then $q \geq 9$; moreover, $p^{t} \leq 3<p^{t+1}$ implies $p^{t}=1$. It follows that $3|g| \leq 3(q+1) \leq q^{2}-q$, and we are done. If $p=3$, then $p^{t} \leq 3<p^{t+1}$ yields $p^{t}=3$ and $|g| \leq 3(q+1)$. Now, $3|g| \leq 9(q+1) \leq q^{2}-q$ if $q \geq 11$; thus we are only left to examine the cases $q=5,8$. In both cases, we get a contradiction with Lemma 2.4

Next, assume that $n>3$. In this case, we will show that $n|g| \leq \frac{q^{n}-(-1)^{n}}{q+1}-1$.

Let us write $q=q_{0}^{p^{m}}$, where $p^{m}=|\Phi|_{p}$. Then, by Lemma 3.1. $|g| \leq p^{t+m} \mid q_{0}^{e / 2}+$ $\left.1\right|_{p} \leq p^{t+m}\left(q_{0}^{e / 2}+1\right) \leq n p^{m}\left(q_{0}^{e / 2}+1\right) \leq n p^{m}\left(q_{0}^{\frac{p-1}{2}}+1\right)($ since $e<p)$.

If $m \geq 1$ and $(n, q) \neq(4,8),(5,8)$, then $n^{2} p^{m}\left(q_{0}^{\frac{p-1}{2}}+1\right)+1 \leq q_{0}^{\left\lfloor\frac{n}{2}\right\rfloor p^{m}}$. Hence $(n|g|+1)(q+1) \leq q_{0}^{\left\lfloor\frac{n}{2}\right\rfloor p^{m}}\left(q_{0}^{p^{m}}+1\right) \leq q_{0}^{n p^{m}}-2$, whence $n|g| \leq \frac{q^{n}-(-1)^{n}}{q+1}-1$. If $L \in\left\{\mathrm{PSU}_{4}(8), \mathrm{PSU}_{5}(8)\right\}$, then $p=3, e=2$ and $t=1$, whence $|g| \leq 3 \cdot|8+1|_{3}=27$. Since $4 \cdot 27 \leq 454=\frac{8^{4}-1}{8+1}-1$ and $5 \cdot 27 \leq 3640=\frac{8^{5}+1}{8+1}-1$, we are done.

Lemma 5.8. Let $L=\operatorname{PSU}_{n}(q)$, with $n>2$ and $q$ odd. Let $g \in \operatorname{Aut} L, g \notin A_{d}$, be a 2-element such that $g^{2} \neq 1$, and set $G=\langle g, L\rangle$. Suppose $(n, q) \notin\{(3,3),(4,3)\}$. Then $g$ is not almost cyclic in any cross-characteristic projective irreducible representation of $G$ that is non-trivial on $L$.

Proof. Set $H=\mathrm{GU}_{n}(q), H_{1}=\mathrm{GL}_{n}\left(q^{2}\right)$ and $q=q_{0}^{2^{m}}$, where $2^{m}=|\Phi|_{2}$. We claim that $\alpha(g) \eta_{2}(G)<\operatorname{dim} \phi$ for every cross-characteristic projective irreducible representation $\phi$ of $G$ non-trivial on $L$, whence the statement by Lemma [2.4] Clearly, we may replace $G$ with $H \cdot \Phi_{2}$. By Lemmas 3.6 and 3.7 $\eta_{2}\left(H_{1} \cdot \Phi_{2}\right)=$ $\eta_{2}\left(H_{1}\right)=2^{t}\left|q^{2}-1\right|_{2}$, where $2^{t} \leq n<2^{t+1}$. Since $H \leq H_{1}$, it follows that $\eta_{2}(H) \leq 2^{t}\left|q^{2}-1\right|_{2} \leq 2 n(q+1)$.

Suppose that $n \geq 5$. Then, by Proposition 2.2, $\alpha(g) \leq n$ and by Lemma 2.8 $\operatorname{dim} \phi \geq \frac{q^{n}-q}{q+1}$. Now, if either $n \geq 7$ or $n=5,6$ and $q \geq 5$, we have $2 n^{2}(q+1)<$ $\frac{q^{n}-q}{q+1}$, and we are done. If $(n, q)=(6,3)$, then we find that $\eta_{2}\left(H_{1}\right)=16$ and $\operatorname{dim} \phi \geq 182$, and we are done. If $(n, q)=(5,3)$, then, again using the GAP package, we find that $\eta_{2}(G)=16$ and moreover, $\alpha(g) \leq 5$ if $|g|=4, \alpha(g) \leq 3$ if $|g|=8$ and $\alpha(g)=2$ if $|g|=16$. Since $\operatorname{dim} \phi \geq 60$, we are done. 
Next, suppose that $n=4$. Then $\operatorname{dim} \phi \geq q^{3}-q^{2}+q-1$ and, by Proposition 2.3 . $\alpha(g) \leq 6$. Since $6 \cdot 8(q+1)<q^{3}-q^{2}+q-1$ if $q \geq 9$, we are left to consider the cases $q=5,7$. For $q=5$ we find that $\eta_{2}(G)=8$, whence the claim, since $\operatorname{dim} \phi \geq 104$. For $q=7$ we find that $\eta_{2}(G) \leq 32$ and $\operatorname{dim} \phi \geq 300$, whence the claim.

Finally, suppose that $n=3$. Then $\operatorname{dim} \phi \geq q^{2}-q, \alpha(g) \leq 3$ and $\eta_{2}\left(H_{1}\right) \leq 4(q+1)$. Since $3 \cdot 4(q+1)<q^{2}-q$ for $q \geq 17$, we are left to consider the cases $q=5,7,9,11,13$. For $q=5$, we find that $\eta_{2}(G)=8$ and $\operatorname{dim} \phi \geq 20$. Moreover, if $|g|=4$, then $\alpha(g) \leq 3$; if $|g|=8$, then $\alpha(g)=2$. Thus in both cases $\phi(g)$ is not almost cyclic.

For $q=7$, we find that $\eta_{2}(G)=16$ and $\operatorname{dim} \phi \geq 42$. If $|g|=4$, or 8 , then $\alpha(g) \leq 3$; if $|g|=16$, then $\alpha(g)=2$. Thus, we are done with the case $q=7$. Next, for $q=9, \eta_{2}(G)=16$ and $\operatorname{dim} \phi \geq 72$; for $q=11, \eta_{2}\left(H_{1}\right)=16$ and $\operatorname{dim} \phi \geq 110$; for $q=13, \eta_{2}\left(H_{1}\right)=2 \cdot 8=16$ and $\operatorname{dim} \phi \geq 156$. It follows that also in these cases, we are done by Lemma 2.4. This ends the proof.

Let $L=\operatorname{PSp}_{2 n}(q)$ and $H=\operatorname{Sp}_{2 n}(q)$, where $q$ is odd and $n>1$. Let $p^{m}=$ $\left|\mathbb{F}_{q}: \mathbb{F}_{r}\right|_{p}$. Recall that $\mathrm{CSp}_{2 n}(q)$ denotes the conformal symplectic group. It is well known that $A_{d}=\operatorname{CSp}_{2 n}(q) / Z$, where $Z$ is the group of scalar matrices in $\operatorname{GL}_{2 n}(q)$. Furthermore, $\left|A_{d}: L\right|=2$, as $q$ is odd.

Lemma 5.9. Let $L$ be as above, with $p$ odd. Let $1 \neq g \in \operatorname{Aut} L$ be a p-element and set $G=\langle g, L\rangle$. Let $\phi$ be a cross-characteristic irreducible projective representation of $G$ such that $\operatorname{dim} \phi>1$. Suppose that $g \notin L$. Then $\phi(g)$ is not almost cyclic.

Proof. By Lemma 3.5. $\eta_{p}($ Aut $L) \leq \eta_{p}(H)$. As $g \notin L$, the assumption $p>2$ implies $q \geq 8$, and hence, as $q$ is odd, $q \geq 27$. By Lemma 2.4, it suffices to show that $2 n|g|<\frac{q^{n}-1}{2} \leq \operatorname{dim} \phi$.

Suppose first that $e$ is odd.

Then $H$ contains a subgroup $H_{1} \cong \mathrm{GL}_{n}(q)$, and $|H|_{p}=\left|H_{1}\right|_{p}$ (again, see [34]). Thus, by the above, $|g| \leq \eta_{p}\left(H_{1}\right)$. The latter, by Lemma 3.2, equals $p^{t}\left|q^{e}-1\right|_{p}$, where $p^{t} e \leq n<p^{t+1} e$. If $n>2$, it follows from Lemma 5.4 that $n|g|<\frac{q^{n}-1}{q-1}-2$. Since $2\left(\frac{q^{n}-1}{q-1}-2\right)<\frac{q^{n}-1}{2}$ for $q \geq 7$, we are done. So, let $n=2$. Then $e=1$ and $|g| \leq \eta_{p}\left(H_{1}\right)=|q-1|_{p}$. Thus $4|g| \leq 4|q-1|_{p}$, and one easily checks that $4|g|<\left(q^{2}-1\right) / 2$ for $q>7$.

Next, let $e$ be even.

Then it is known that $\mathrm{Sp}_{2 n}(q)$ contains a Sylow $p$-subgroup of $\mathrm{GL}_{2 n}(q)$ (again, see [34]). Write $2 n=a e+b$, where $0 \leq b<e$ and, as usual, let $q=q_{0}^{p^{m}}$, where $p^{m}=|\Phi|_{p}$. It is easy to observe that $\left|\mathrm{GL}_{2 n}(q)\right|_{p}=\left|\mathrm{GL}_{a e}(q)\right|_{p}$. Therefore $\left|\mathrm{GL}_{a e}(q)\right|_{p}=\left|\operatorname{Sp}_{a e}(q)\right|_{p}$, whence $\left|\operatorname{Sp}_{2 n}(q)\right|_{p}=\left|\operatorname{Sp}_{a e}(q)\right|_{p}$. Thus $|g| \leq \eta_{p}\left(\operatorname{GL}_{a e}(q)\right)$.

Suppose first that $2 n=a e$. Observe that, by Lemma 3.1(1), $e=e_{p}(q)=$ $e_{p}\left(q_{0}^{p^{m}}\right)=e_{p}\left(q_{0}\right)$, and hence $p$ divides $q_{0}^{e}-1$. It then follows, by Lemmas 3.1 (2) and 3.2, that $|g| \leq \eta_{p}\left(\mathrm{GL}_{a e}(q)\right)=p^{t+m}\left|q_{0}^{e}-1\right|_{p}$, where $p^{t} \leq a<p^{t+1}$. We wish to show that $a e|g| \leq 2 n p^{t+m}\left|q_{0}^{e}-1\right|_{p} \leq \frac{4 n^{2}}{e} p^{m}\left(q_{0}^{e}-1\right)<\frac{q^{a e} / 2}{2}-1$. To this purpose, it suffices to show that $4 n^{2} p^{m}\left(q_{0}^{2 n}-1\right)<\frac{q_{0}^{n p^{m}}-1}{2}$. Now, $8 n^{2} p^{m} q_{0}^{2 n}<q_{0}^{n p^{m}}$ holds for any $p \geq 3$, any $n \geq 2$ and any $q_{0} \geq 3$, unless $p=3, m=1$ and one of the following occurs:

(i) $q_{0}=3$ and $n \leq 6$;

(iii) $q_{0}=7$ and $n=2$; (ii) $q_{0}=5$ and $n \leq 3$

(iv) $\quad q_{0}=9$ and $n=2$. 
As $p \nmid q$, cases $(i)$ and $(i v)$ are ruled out. On the other hand, in all the other cases, we still have $2 n|g| \leq \frac{q^{n}-1}{2}$. Namely, in case $(i i)$, for $n=2$ we have $2 n|g|=4|g| \leq$ $4 \eta_{3}\left(\mathrm{GL}_{4}\left(5^{3}\right)\right)=36<\frac{125^{2}-1}{2}$, and for $n=3$ we have $6|g| \leq 6 \eta_{3}\left(\mathrm{GL}_{6}\left(5^{3}\right)\right)=162<$ $\frac{125^{3}-1}{2}$. In case $($ iii $)$ we have $2 n|g|=4|g| \leq 4 \eta_{3}\left(\mathrm{GL}_{4}\left(7^{3}\right)\right)=108<\frac{343^{2}-1}{2}$.

Finally, suppose $2 n>a e$. Then $2 n|g|=\frac{2 n}{a e} a e|g| \leq \frac{2 n}{a e} \cdot \frac{\left(q^{a e / 2}-1\right)}{2}<\frac{q^{n}-1}{2}$ for every $n \geq 2$.

Lemma 5.10. Let $G=\operatorname{Sp}_{2 n}(q)$ and $H=\operatorname{CSp}_{2 n}(q)$, where $q$ is odd.

(1) $G$ has two inequivalent irreducible $\mathbb{F}$-representations of degree $\left(q^{n}-1\right) / 2$; they do not extend to $H$.

(2) Let char $\mathbb{F} \neq 2$. Then $G$ has two inequivalent irreducible $\mathbb{F}$-representations of degree $\left(q^{n}+1\right) / 2$. They do not extend to $H$.

(3) If $\phi$ is an irreducible $\mathbb{F}$-representation of $H$ with $\operatorname{dim} \phi>1$, then $\operatorname{dim} \phi \geq$ $q^{n}-1$.

Proof. (1) and (2): It is well known that, over the complex numbers, $G$ has two inequivalent irreducible representations of degree $\left(q^{n}-1\right) / 2$ and two inequivalent irreducible representations of degree $\left(q^{n}+1\right) / 2$ (see [9]). Moreover, it follows from [9, Proposition 1.4] that the representations of equal degrees can be obtained from each other by twisting by an involution $h \in H$, and hence none of them extends to $H$. The reductions modulo an odd prime distinct from $\ell$ of these representations remain irreducible and inequivalent (see [33]). As for the representations of degree $\left(q^{n}-\right.$ 1) $/ 2$ the reduction modulo 2 remains irreducible, whereas the reduction modulo 2 of the representations of degree $\left(q^{n}+1\right) / 2$ has a composition factor of degree $\left(q^{n}-1\right) / 2$. This implies that the irreducible $\mathbb{F}$-representations of $G$ do not extend to $H$. Moreover, it is clear that $H$ has an irreducible $\mathbb{F}$-representation of degree $q^{n}-1$, whose restriction to $G$ splits into two inequivalent irreducible representations of $G$ of degree $\left(q^{n}-1\right) / 2$.

(3): By [13, Theorem 2.1], it is known that, if $\tau$ is an irreducible $\mathbb{F}$-representation of $G$ of degree greater that $\left(q^{n}+1\right) / 2$, then $\operatorname{dim} \tau \geq\left(q^{n}-1\right)\left(q^{n}-q\right) / 2(q+1)$. It follows from Clifford's theorem that the same is true for $\phi$, provided $\operatorname{dim} \phi>q^{n}+1$.

Now, suppose $\operatorname{dim} \phi \leq q^{n}+1$. Again by [13. Theorem 2.1], it follows from Clifford's theorem that the composition factors of $\left.\phi\right|_{G}$ are of degree $\left(q^{n} \pm 1\right) / 2$. On the other hand, by the above, $\operatorname{dim} \phi=q^{n} \pm 1$. Taking into account that, if char $\mathbb{F}=2$, there are no irreducible representation of $G$ of degree $\left(q^{n}+1\right) / 2$, item (3) follows.

Lemma 5.11. Let $L=\operatorname{PSp}_{2 n}(q)$, where $n \geq 2$ and $q$ is odd. Let $1 \neq g \in$ Aut $L$ be a 2-element such that $g^{2} \neq 1$, and let $\bar{G}=\langle g, L\rangle$. Suppose that $(2 n, q) \neq$ $(4,3)$. Then $g$ is not almost cyclic in any cross-characteristic projective irreducible representation $\phi$ of $G$ that is non-trivial on $L$.

Proof. Let $G_{1}=\operatorname{CSp}_{2 n}(q) \cdot \Phi$. Then $G_{1} \subseteq \mathrm{GL}_{2 n}(q) \cdot \Phi$. By Lemmas 3.6] and 3.7] we have $\eta_{2}($ Aut $L) \leq \eta_{2}\left(G_{1}\right) \leq \eta_{2}\left(\mathrm{GL}_{2 n}(q) \cdot \Phi\right)=\eta_{2}\left(\mathrm{GL}_{2 n}(q)\right) \leq 2^{t+1}(q+1)$, where $2^{t} \leq n<2^{t+1}$. For $n>2$, we get that $\phi(g)$ is not almost cyclic by Lemma 2.4 once we show that $2 n|g|<\frac{q^{n}-1}{2}$. Now, $2 n|g| \leq 2 n \cdot 2^{t+1}(q+1) \leq$ $4 n^{2}(q+1)$ and $4 n^{2}(q+1)<\frac{q^{n}-1}{2}$ for all $n \geq 3$ and $q$ odd, unless $(n, q) \in$ $\{(3,3),(3,5),(3,7),(4,3),(4,5),(5,3),(6,3)\}$. For $L$ as listed in the Table 1 by Lemma 2.4. we conclude that $\phi(g)$ is not almost cyclic. 
If $L=\operatorname{PSp}_{6}(3)$, then $|g| \leq 8$ and $\operatorname{dim} \phi \geq 13$. If $|g|=4$, then $\alpha(g) \leq 3$, and hence, by Lemma 2.4 $\phi(g)$ is not almost cyclic. If $|g|=8$, then $\alpha(g)=2$; hence we only need to examine the representations $\phi$ such that $13 \leq \operatorname{dim} \phi \leq 14$. Using the GAP package we see that $\phi(g)$ is not almost cyclic.

If $L=\operatorname{PSp}_{12}(3)$, we have $|g| \leq \eta_{2}\left(\mathrm{GL}_{12}(3)\right)=32, \operatorname{dim} \phi \geq 364$ and $\alpha(g) \leq 12$. Thus, if $|g|=4,8,16$, we are done by Lemma 2.4. If $|g|=32$, then $g \notin L$ (since $\left.\eta_{2}\left(\operatorname{PSp}_{12}(3)\right)=16\right)$. So, we can refine the bound using Lemma 5.10, and again we are done by Lemma 2.4 .

Suppose now that $n=2$ and $q \geq 5$. Then it is enough to show that $5|g| \leq \frac{q^{2}-1}{2}$ (see Proposition 2.3 (6)). By the above $5|g| \leq 5 \cdot 2^{2}(q+1)$, and $20(q+1)<\frac{q^{2}-1}{2}$ for all $q>41$. Assume $q \leq 41$. Then we may refine the previous bound, obtaining that $5|g| \leq 5 \eta_{2}\left(\mathrm{GL}_{4}(q)\right) \leq \frac{q^{2}-1}{2}$ unless $q=5,7,9,11,17,31$. In these exceptional cases we get the following:

\begin{tabular}{c|c|c|c||c|c|c|c}
$L$ & $|g|$ & $\alpha(g)$ & $\operatorname{dim} \phi \geq$ & $L$ & $|g|$ & $\alpha(g)$ & $\operatorname{dim} \phi \geq$ \\
\hline $\mathrm{PSp}_{4}(5)$ & $=4$ & $\leq 3$ & 12 & $\operatorname{PSp}_{4}(9)$ & $=16$ & $=2$ & 40 \\
$\operatorname{PSp}_{4}(5)$ & $=8$ & $=2$ & 12 & $\operatorname{PSp}_{4}(11)$ & $\leq 8$ & $\leq 3$ & 60 \\
$\operatorname{PSp}_{4}(7)$ & $\leq 8$ & $\leq 3$ & 24 & $\operatorname{PSp}_{4}(17)$ & $\leq 32$ & $\leq 3$ & 144 \\
$\operatorname{PSp}_{4}(7)$ & $=16$ & $=2$ & 24 & $\operatorname{PSp}_{4}(31)$ & $\leq 64$ & $\leq 5$ & 480 \\
$\operatorname{PSp}_{4}(9)$ & $\leq 8$ & $\leq 3$ & 40 & & & &
\end{tabular}

Hence, by Lemma 2.4 we are only left to consider the following instances: (i) $L=\mathrm{PSp}_{4}(5)$ and $|g|=8$. Then $g \notin L$, whence $\operatorname{dim} \phi \geq 24$. Thus, by Lemma 2.4. $\phi(g)$ is not almost cyclic.

(ii) $L=\mathrm{PSp}_{4}(7)$ and $|g|=16$. In this case $g \notin L$, whence $\operatorname{dim} \phi \geq 48$ (again by Lemma 5.10), which implies, by Lemma 2.4, that $\phi(g)$ is not almost cyclic.

5.2.2. Case $p \mid q$. In this subsection we examine the case where $p$ divides $q$. Although, for $p$ odd, we might refer to the earlier paper [5], the approach chosen in the present paper (for all $p$ ) appears to be more efficient than in [5] (and may lead to a shorter proof of the results in [5]).

First, let $H=\mathrm{GL}_{n}(q)$ and let $\Phi$ be the group of field automorphisms of $H$. Suppose that $t$ and $m$ are such that $p^{t}<n \leq p^{t+1}$ and $|\Phi|_{p}=p^{m}$. Write $q=q_{0}^{p^{m}}$. Observe that $\eta_{p}(H)=p^{t+1}$. Indeed, $p^{t+1}$ is exactly the order of a unipotent element of $\mathrm{GL}_{n}(q)$ consisting of single Jordan block of size $n$. Furthermore, note that $\eta_{p}(H)=p^{t+1}$ also for $H=\mathrm{SL}_{n}(q), \mathrm{GU}_{n}(q)$ and $\mathrm{SU}_{n}(q)$.

Next, we need the following arithmetical lemma.

Lemma 5.12. Let $p$ be a prime and let $q$ be a p-power. Let $n$ be an integer greater than 4, and suppose that $t, m$ and $q_{0}$ are defined as above. If $p=2$, then $n 2^{t+m+2} \leq \frac{q^{n}-q}{q+1}$ unless $q=2$ and $5 \leq n \leq 9$. If $p$ is odd, then $n p^{t+m+1} \leq \frac{q^{n}-q}{q+1}$. Furthermore, $n 2^{t+m+2} \leq \frac{q^{n}-1}{q-1}-2$, unless $q=2$ and $n=5,6$.

Proof. Since $p^{t}<n \leq p^{t+1}$, we have $n p^{t+m+2} \leq p^{2 t+m+3}$ and $\frac{q^{n}-q}{q+1} \geq q^{n-2}=$ $q_{0}^{p^{m}(n-2)} \geq p^{p^{m}\left(p^{t}-1\right)}$. Hence, it suffices to prove that $p^{2 t+m+3} \leq p^{p^{m}\left(p^{t}-1\right)}$, which is equivalent to prove that $2 t+m+3 \leq p^{m}\left(p^{t}-1\right)$. Notice that $2 t+m+3 \leq 2^{m}\left(2^{t}-1\right)$ holds unless one of the following cases occurs: $(i) t=0 ;(i i) t=1$ and $m=0,1,2$; (iii) $t=2$ and $m=0,1 ;(i v) t=3$ and $m=0$. Direct computations in each case yield the statement. 
Lemma 5.13. Let $L \in\left\{\operatorname{PSL}_{n}(q), \operatorname{PSU}_{n}(q)\right\}$, where $n \geq 5$. Let $g \in$ Aut $L$ be a $p$-element such that $g^{2} \neq 1$ and let $G=\langle g, L\rangle$. Then $\phi(g)$ is almost cyclic for some cross-characteristic projective irreducible representation $\phi$ of $G$ that is non-trivial on $L$ if and only if $G=\operatorname{PSU}_{5}(2) .2, \operatorname{dim} \phi=10$ and $|g|=16$.

Proof. Recall (see Lemma 2.8) that $\operatorname{dim} \phi \geq \frac{q^{n}-1}{q-1}-2$ if $L=\operatorname{PSL}_{n}(q)$, whereas $\operatorname{dim} \phi \geq \frac{q^{n}-q}{q+1}$ if $L=\operatorname{PSU}_{n}(q)$ and $n$ is odd, and $\operatorname{dim} \phi \geq \frac{q^{n}-1}{q+1}$ if $L=\operatorname{PSU}_{n}(q)$ and $n$ is even. Also, notice that $\frac{q^{n}-q}{q+1} \leq \frac{q^{n}-1}{q+1} \leq \frac{q^{n}-1}{q-1}-2$.

If $p \geq 3$, then $\eta_{p}(G) \leq p^{t+m+1}$, where $t$ and $m$ are defined as above. By Lemma 5.12 we get $n|g| \leq n p^{t+m+1} \leq \operatorname{dim} \phi$. Thus $\phi(g)$ is not almost cyclic, by Lemma 2.4. If $p=2$, then $\eta_{2}(G) \leq 2^{t+1} \cdot 2^{t+m+2}$. Likewise, we may apply Lemma 5.12 obtaining that $\phi(g)$ is not almost cyclic, unless $L$ is one of the following groups: $\mathrm{PSL}_{5}(2), \mathrm{PSU}_{5}(2), \mathrm{PSL}_{6}(2), \mathrm{PSU}_{6}(2), \mathrm{PSU}_{7}(2), \mathrm{PSU}_{8}(2)$ and $\mathrm{PSU}_{9}(2)$. Thus, we need to examine these groups in detail. Moreover, in order to bound efficiently $\alpha(g)$ we make use of the package GAP whenever necessary.

1) $L=\mathrm{PSL}_{5}(2)$. In this case $|g| \leq 16$ and $\operatorname{dim} \phi \geq 29$. If $|g|=4$, then $\alpha(g) \leq 3$; if $|g|=8$, then $\alpha(g)=2$. Thus, if $|g| \leq 8, \phi(g)$ is not almost cyclic, by Lemma 2.4 If $|g|=16$, then $\alpha(g)=2$. This means that we have to examine the representations $\phi$ such that $29 \leq \operatorname{dim} \phi \leq 30$. Using the GAP package, again we see that $\phi(g)$ is not almost cyclic.

2) $L=\operatorname{PSU}_{5}$ (2). In this case $|g| \leq 16$ and $\operatorname{dim} \phi \geq 10$. If $|g|=4$, then $\alpha(g) \leq 3$. Thus $\phi(g)$ is not almost cyclic by Lemma 2.4. If $|g|=8$, then $\alpha(g)=2$, and hence we need to examine the representations $\phi$ such that $10 \leq \operatorname{dim} \phi \leq 14$. If $|g|=16$, then $\alpha(g)=2$ and we need to examine the representations $\phi$ such that $10 \leq \operatorname{dim} \phi \leq 30$. Using the GAP package, we see that $\phi(g)$ is almost cyclic if and only if $G=\mathrm{PSU}_{5}(2) \cdot 2, \operatorname{dim} \phi=10$ and $|g|=16$, as claimed.

3) $L=\operatorname{PSL}_{6}(2)$. In this case $|g| \leq 16, \alpha(g) \leq 3$ and $\operatorname{dim} \phi \geq 61$. Thus, by Lemma 2.4 we conclude that $\phi(g)$ is not almost cyclic.

4) $L=\mathrm{PSU}_{6}(2)$. In this case $|g| \leq 16$ and $\operatorname{dim} \phi \geq 21$. If $|g|=8$ or 16 , then $\alpha(g)=2$, whereas if $|g|=4$, then $\alpha(g) \leq 3$. Thus, if $|g|=4$ or 8 , by Lemma $2.4 \phi(g)$ is not almost cyclic. If $|g|=16(g \notin L)$, we need to examine the representations $\phi$ such that $21 \leq \operatorname{dim} \phi \leq 30$. Using the GAP package, we find that $\phi(g)$ is never almost cyclic.

5) $L=\operatorname{PSU}_{7}(2)$. In this case $|g| \leq 16$ and $\operatorname{dim} \phi \geq 42$. If $|g|=4$, then $\alpha(g) \leq 4$, whereas, if $|g|=8$ or 16 , then $\alpha(g)=2$. In both cases, by Lemma 2.4, $\phi(g)$ is not almost cyclic.

6) $L=\mathrm{PSU}_{8}(2)$. In this case $|g| \leq 16, \alpha(g) \leq 4$ and $\operatorname{dim} \phi \geq 85$. By Lemma 2.4. $\phi(g)$ is not almost cyclic.

7) $L=\operatorname{PSU}_{9}(2)$. In this case $|g| \leq 32$ and $\operatorname{dim} \phi \geq 170$. If $|g| \leq 16$, then $\phi(g)$ is not almost cyclic by Lemma 2.4 If $|g|=32$, then we may consider $g^{16}$. The latter belongs to $L$, and $L$ is generated by 9 conjugates of $g^{16}$. By Lemma 2.4. $\operatorname{dim} \phi \leq 9 d$, where $d$ equals the dimension of an eigenspace of $\phi\left(g^{16}\right)$. Suppose that $\phi(g)$ is almost cyclic. Then it readily follows that either 1 or -1 appears as an eigenvalue of $\phi\left(g^{16}\right)$ with multiplicity $\leq 16$. Thus we are led to conclude that $\operatorname{dim} \phi \leq 9 d \leq 9 \cdot 16<170$, a contradiction.

Lemma 5.14. Let $L \in\left\{\mathrm{PSL}_{3}(q), \mathrm{PSL}_{4}(q)\right\}$, with $L \neq \mathrm{PSL}_{3}(2), \mathrm{PSL}_{3}(4), \mathrm{PSL}_{4}(2)$. Let $g \in$ Aut $L$ be a p-element such that $g^{2} \neq 1$ and let $G=\langle g, L\rangle$. Then $g$ is not 
almost cyclic in any cross-characteristic projective irreducible representation $\phi$ of $G$ that is non-trivial on $L$.

Proof. Let $L=\operatorname{PSL}_{3}(q)$. If $q=3$, then $|g|=3, \alpha(g) \leq 3$ and $\operatorname{dim} \phi \geq 11$, whence $11 \leq 3(3-1)=6$. Thus $\phi(g)$ cannot be almost cyclic. Now, assume that $q \geq 5$.

Let $t$ and $m$ be defined as above and suppose that $p>2$. Then $n=3$ implies $t=0$, whence $\eta_{p}(G) \leq p^{m+1}$. Therefore $3 \eta_{p}(G) \leq 3 p^{m+1} \leq p^{m+2}$, whilst $\operatorname{dim} \phi \geq$ $q^{2}+q-1 \geq q^{2} \geq p^{2 p^{m}}$. Thus it is enough to show that $m+2 \leq 2 \cdot 3^{m} \leq 2 p^{m}$. This clearly holds for all $m \geq 0$. If $p=2$, then $\eta_{2}(G) \leq 2^{m+3}$. Hence (see Proposition (2.3) we need to show that $4 \eta_{2}(G) \leq 2^{m+5} \leq 2^{2^{m+1}} \leq q^{2}+q-1$. Now, $m+5 \leq 2^{m+1}$ provided $m>1$. For $m=0,1$, we get the inequality $4 \cdot 2^{m+3} \leq 64 \leq q^{2}+q-1$, which holds for any even $q>4$.

Next, let $L=\mathrm{PSL}_{4}(q)$. If $q=3$, then $|g| \leq 9$ and $\operatorname{dim} \phi \geq 26$. If $|g|=3$, then $\alpha(g) \leq 4$, whereas, if $|g|=9$, then $\alpha(g)=2$. In both cases, $\phi(g)$ cannot be almost cyclic, by Lemma 2.4. Now, assume that $q \geq 5$.

If $p>3$, then $\eta_{p}(G) \leq p^{m+1}$. Hence $4 \eta_{p}(G) \leq 4 p^{m+1} \leq p^{m+2} \leq p^{3 p^{m}} \leq$ $q^{3}+q^{2}+q-1 \leq \operatorname{dim} \phi$, and we are done. If $p=3$, then $\eta_{3}(G) \leq 3^{m+2}$, and hence $4 \eta_{3}(G) \leq 4 \cdot 3^{m+2} \leq 3^{m+4} \leq 3^{3^{m+1}} \leq \operatorname{dim} \phi$ for all $m \geq 1$. If $m=0$ (and $q>3$ ), we still have $4 \eta_{3}(G) \leq 36 \leq q^{3} \leq \operatorname{dim} \phi$. Finally, let $p=2$. Then $\eta_{2}(G) \leq 2^{m+3}$. By Proposition $2.3 \alpha(g) \leq 6$, whence $6 \eta_{2}(G) \leq 2^{m+6} \leq 2^{3 \cdot 2^{m}} \leq \operatorname{dim} \phi$ for all $m \geq 2$. Furthermore, if $m=0,1$ (and $q>4$ ), then $6 \eta_{2}(G) \leq 96 \leq 8^{3} \leq \operatorname{dim} \phi$.

Finally, let $L=\mathrm{PSL}_{4}(4)$. In this case $|g|=4,8, \alpha(g) \leq 3$ and $\operatorname{dim} \phi \geq 83$, and hence $\phi(g)$ cannot be almost cyclic, by Lemma 2.4 .

Lemma 5.15. Let $L \in\left\{\operatorname{PSU}_{3}(q), q>4, \operatorname{PSU}_{4}(q), q>3\right\}$. Let $g \in$ Aut $L$ be a p-element such that $g^{2} \neq 1$ and let $G=\langle g, L\rangle$. Then $g$ is not almost cyclic in any cross-characteristic projective irreducible representation $\phi$ of $G$ that is non-trivial on $L$.

Proof. Let $L=\operatorname{PSU}_{3}(q)$. If $p>2$, then $\eta_{p}(G) \leq p^{m+1}$. Since $3 \eta_{p}(G) \leq 3 p^{m+1}$ and $\operatorname{dim} \phi \geq q^{2}-q \geq 3 q \geq 3 p^{p^{m}}$, it suffices to show that $m+1 \leq p^{m}$, which clearly holds for all $m \geq 0$. If $p=2$, then $\eta_{2}(G) \leq 2^{m+3}$. Hence, it will be enough to show that $4 \eta_{2}(G) \leq 2^{m+5} \leq 2^{1+2^{m}} \leq 2 q \leq q^{2}-q$. Now, $m+4 \leq 2^{m}$ provided $m \geq 3$. If $0 \leq m \leq 2$, then $4 \eta_{2}(G) \leq 128 \leq q^{2}-q$ for all $q \geq 16$. If $q=8$, then $4 \eta_{2}(G)=32 \leq \operatorname{dim} \phi$. Therefore, we are done.

Now, let $L=\operatorname{PSU}_{4}(q)$. If $p>3$, then $\eta_{p}(G) \leq p^{m+1}$. Whence $4 \eta_{p}(G) \leq$ $4 p^{m+1} \leq 4 p^{2 p^{m}} \leq 4 q^{2} \leq q^{3}-q^{2}+q-1 \leq \operatorname{dim} \phi$ (note that $m+1 \leq 2 p^{m}$ for all $m \geq 0)$. If $p=3$, then $\eta_{3}(G) \leq 3^{m+2}$. Hence, $4 \eta_{3}(G) \leq 4 \cdot 3^{m+2} \leq 4 \cdot 3^{2 \cdot 3^{m}} \leq \operatorname{dim} \phi$ for all $m \geq 0$. If $p=2$, then $\eta_{2}(G) \leq 2^{m+3}$. It follows that $6 \eta_{2}(G) \leq 6 \cdot 2^{m+3} \leq$ $6 \cdot 2^{2^{m+1}} \leq 6 q^{2} \leq \operatorname{dim} \phi$ holds for all $m \geq 1$. If $m=0$ (and $q>4$ ), then $6 \eta_{2}(G) \leq 48 \leq q^{3}-q^{2}+q-1$.

Finally, let $L=\mathrm{PSU}_{4}(4)$. In this case $\eta_{2}(G)=16, \alpha(g)=2$ and $\operatorname{dim} \phi \geq 51$; hence $\phi(g)$ cannot be almost cyclic, by Lemma 2.4

Lemma 5.16. Let $L=\operatorname{PSp}_{2 n}(q)$, where $n \geq 2$ and $q$ is odd. Let $1 \neq g \in$ Aut $L$ be a p-element and let $G=\langle g, L\rangle$. Let $\phi$ be a cross-characteristic projective irreducible representation $G$ that is non-trivial on $L$. Then $\phi(g)$ is almost cyclic if and only if $L=\mathrm{PSp}_{4}(3)$ and the following occurs:

(1) $\ell \neq 2,3: g=3 a, 3 b$ and $\operatorname{dim} \phi=4 ; g=3 c$ and $\operatorname{dim} \phi=6 ; g=3 d$ and $\operatorname{dim} \phi=4,5 ;|g|=9$ and $\operatorname{dim} \phi=4,5,6$; 
(2) $\ell=2: g=3 a, 3 b$ and $\operatorname{dim} \phi=4 ; g=3 c$ and $\operatorname{dim} \phi=6 ; g=3 d$ and $\operatorname{dim} \phi=4 ;|g|=9$ and $\operatorname{dim}=4,6$.

Proof. First, note that $\eta_{p}(G) \leq p^{t+m+1}$, where $p^{t}<2 n \leq p^{t+1}$. Thus, we aim to show that $2 n \eta_{p}(G) \leq 2 n p^{t+m+1} \leq \frac{q^{n}-1}{2}$, that is, $4 n p^{t+m+1} \leq q^{n}-1$. Now, $4 n p^{t+m+1} \leq 2 p^{2 t+m+2} \leq p^{2 t+m+3}-1$ and $q^{n}-1 \geq p^{n p^{m}}-1$. Therefore, it will suffice to consider the inequality $2 t+m+3 \leq n p^{m}$.

If $g \in L$, we may refer to [5]. In particular, by [5, Theorem 1.1 and Lemma 4.2], $\phi(g)$ is almost cyclic if and only if $L=\mathrm{PSp}_{4}(3)$ and (1) or (2) holds. So, suppose that $g \notin L$. This implies in particular that we may assume $m>0$.

If $t \geq 1$, then $2 t+m+3 \leq p^{t+m-1} \leq n p^{m}$, unless $t=m=1$ and $p=3,5$. For $p=3$, we get $3<2 n \leq 9$ and $2 n \eta_{3}(G) \leq 54 n \leq \frac{3^{3 n}-1}{2}$. For $p=5$, we get $5<2 n \leq 25$ and $2 n \eta_{5}(G) \leq 250 n \leq \frac{5^{5 n}-1}{2}$. Thus we are done.

Finally, suppose that $t=0$, i.e. $2 n \leq p$. In this case, we have to consider the inequality $2 n \eta_{p}(G) \leq p^{m+2} \leq \frac{p^{n p^{m}}-1}{2}$. Since $2 p^{m+2} \leq p^{m+3}-1 \leq p^{n p^{m}}-1$ is equivalent to $m+3 \leq n p^{m}$, we only need to observe that $m+3 \leq 2 \cdot 3^{m}$ holds for all $m \geq 1$, thus proving the statement.

\section{Some LOW-DIMENSIONAL ClASSICAL GROUPS}

In this section we deal with the low-dimensional simple groups $L$ which were left out in either or both the two previous sections. These groups, which need a separate treatment, are the following: $\mathrm{PSL}_{3}(2), \mathrm{PSL}_{3}(4), \mathrm{PSL}_{4}(2), \mathrm{PSU}_{3}(3), \mathrm{PSU}_{4}(2) \cong$ $\mathrm{PSp}_{4}(3), \mathrm{PSU}_{4}(3)$. Observe that, with the exception of $\mathrm{PSU}_{3}(3)$ they all have an exceptional Schur multiplier (by this, we mean that the order of the multiplier is divisible by the defining characteristic of the group in question). As always, $G=\langle g, L\rangle$, where $g \in$ Aut $L$. When needed, we use the notation of the GAP package to denote $G$ and the conjugacy class of $g$. Also, in view of Lemma 2.10. we may assume $g^{2} \neq 1$.

6.1. $L=\operatorname{PSL}_{3}(2) \cong \operatorname{PSL}_{2}(7)$. Here $|g| \in\{3,4,7,8\}$ and $\alpha(g)=2$. Using the GAP package, we find that $\phi(g)$ is almost cyclic if and only if one of the following occurs:

- $|g|=3$ and either $\ell \neq 7$ and $\operatorname{dim} \phi=3,4$, or $\ell=7$ and $\operatorname{dim} \phi=2,3,4$;

- $|g|=4$ and either $\ell \neq 7$ and $\operatorname{dim} \phi=3,4$, or $\ell=7$ and $\operatorname{dim} \phi=2,3,4,5$;

- $|g|=7$ and either $\ell \neq 3,7$ and $\operatorname{dim} \phi=3,4,6,7,8$, or $\ell=3$ and $\operatorname{dim} \phi=$ $3,4,6,7$, or $\ell=7$ and $\operatorname{dim} \phi=2,3,4,5,6,7$;

- $|g|=8$ and either $\ell \neq 7$ and $\operatorname{dim} \phi=6,7,8$, or $\ell=7$ and $\operatorname{dim} \phi=$ $2,3,4,5,6,7$.

6.2. $L=\mathrm{PSL}_{3}(4)$. Here $|g| \in\{3,4,5,7,8\}$. If $g \notin L$ and has order 3 , then $\alpha(g) \leq 3$; otherwise $\alpha(g)=2$.

Suppose first that $g$ is a 2-element. Then, using the GAP and MAGMA packages we find that $\phi(g)$ is almost cyclic if and only if one of the following occurs:

- $\ell \neq 3,7:|g|=8$ and either $G=L .2_{1}$ and $\operatorname{dim} \phi=6$ or $G=L .2_{3}$ and $\operatorname{dim} \phi=8$

- $\ell=3:|g|=4, G=L$ and $\operatorname{dim} \phi=4 ;|g|=8$ and either $G=L .2_{1}$ and $\operatorname{dim} \phi=6$, or $G=L .2_{2}$ and $\operatorname{dim} \phi=4,6$, or $G=L .2_{3}$ and $\operatorname{dim} \phi=6,8$;

- $\ell=7:|g|=8$ and either $G=L .2_{1}$ and $\operatorname{dim} \phi=6$, or $G=L .2_{3}$ and $\operatorname{dim} \phi=8$, or $g \in 8 a$ and $\operatorname{dim} \phi=10$. 
Next, suppose that $|g|=3$. Then, again using the GAP and MAGMA packages we find that $\phi(g)$ is almost cyclic if and only if:

- $\ell=3, g \in L$ and $\operatorname{dim} \phi=4$.

Finally, suppose that $|g|=5,7$. Here in principle we have to examine representations of dimension 4, 6, 8 and 10, where 4-dimensional representations only occur for $\ell=3$. Furthermore, the Sylow 5 -subgroups as well as the Sylow 7 -subgroups are cyclic; hence, when $\ell=5$ and $\ell=7$, respectively, we can refer to 6, Lemma 2.13 and Corollary 2.14]. As a result, we get that $\phi(g)$ is almost cyclic if and only if one of the following occurs:

- $|g|=5$ and either $\ell=3$ and $\operatorname{dim} \phi=4$, or $\operatorname{dim} \phi=6$;

- $|g|=7$ and either $\ell=3$ and $\operatorname{dim} \phi=4$, or $\operatorname{dim} \phi=6,8$.

6.3. $L=\mathrm{PSL}_{4}(2)$. Here $|g| \in\{3,4,5,7,8\}$ and either $\operatorname{dim} \phi \in\{7,8,13,14\}$ or $\operatorname{dim} \phi \geq 16$. If $|g|=3$, then $g \in L$ and $\alpha(g) \leq 4$, and hence we only have to examine the representations $\phi$ of dimension 7 and 8. If $|g|=4$ and $g \notin L$, then $\alpha(g) \leq 3$, and hence we only have to examine the representations $\phi$ such that $7 \leq \operatorname{dim} \phi \leq 9$. In all the other cases, $\alpha(g)=2$. Using the GAP package, we find that $\phi(g)$ is almost cyclic if and only if one of the following occurs:

- $\operatorname{dim} \phi=7$ and either $g \in 3 a, 5$, or $|g|=4$ and $g \notin L$;

- $\operatorname{dim} \phi=7,8$ and $|g|=7,8$.

6.4. $L=\mathrm{PSU}_{3}(3)$. Using the GAP package, we find that $\alpha(g)=2$ for $|g|=7,8$ and $\alpha(g) \leq 3$ for $|g|=3,4$. Suppose first that $p \neq 3$. The following holds.

If $G=L$, then $\phi(g)$ is almost cyclic if and only if:

- $|g|=7,8, \operatorname{dim} \phi=6,7$ and $\ell \neq 2,3$;

- $|g|=7,8, \operatorname{dim} \phi=6$ and $\ell=2$.

If $G=L .2$, then $p=2$ and $\phi(g)$ is almost cyclic if and only if:

- $|g|=8, \operatorname{dim} \phi=6,7$ and $\ell \neq 2,3$;

- $|g|=8, \operatorname{dim} \phi=6$ and $\ell=2$.

Next, assume that $p=3$. In this case $|g|=3, \alpha(g) \leq 3$ and $\operatorname{dim} \phi \geq 6$. So, we only need to examine the representations $\phi$ of degree 6 . Note that $g \in L$. Again, using the GAP package, we obtain that $\phi(g)$ is not almost cyclic.

6.5. $L=\mathrm{PSU}_{3}(4)$. This group was left out by assumption in Lemma 5.15. In this case $\eta_{2}(G)=16, \alpha(g)=2$ and $\operatorname{dim} \phi \geq 12$. If $|g|=4$, then $\phi(g)$ cannot be almost cyclic, by Lemma 2.4. If $|g|=8$, we only need to examine the representations $\phi$ such that $12 \leq \operatorname{dim} \phi \leq 14$. If $|g|=16$, we only need to examine the representations $\phi$ such that $12 \leq \operatorname{dim} \phi \leq 30$. Using the GAP package, we find that $\phi(g)$ is almost cyclic if and only if $|g|=16$ and $\operatorname{dim} \phi=12$.

6.6. $L=\mathrm{PSU}_{4}(2) \cong \mathrm{PSp}_{4}(3)$. In this case, $|g| \in\{3,4,5,8,9\}$. If $|g|=3$, then $g \in L$ and $\alpha(g) \leq 4$. If $|g|=4$ and $g \in L$, then $\alpha(g)=2$ (in which case we only have to examine the representations $\phi$ such that $4 \leq \operatorname{dim} \phi \leq 6)$. If $|g|=4$ and $g \notin L$, then $\alpha(g) \leq 3$ (in which case we only have to examine the representations $\phi$ such that $4 \leq \operatorname{dim} \phi \leq 9$ ). If $|g|=8$, then $g \notin L$ and $\alpha(g)=2$ (hence we only have to examine the representations $\phi$ such that $4 \leq \operatorname{dim} \phi \leq 14$ ). Finally, if $|g|=5,9$, then $\alpha(g)=2$. Using the GAP package we find that $\phi(g)$ is almost cyclic if and only if one of the following cases occurs:

- $G=L$ and 
(a) $\ell \neq 3, g \in 3 a, 3 b, 3 d$ or $|g|=4,5,9$ and $\operatorname{dim} \phi=4$;

(b) $\ell=3, g \in 3 a, 3 b, 4 a$ or $|g|=5,9$ and $\operatorname{dim} \phi=4$;

(c) $\ell \neq 2,3, g \in 3 d, 4 b$ or $|g|=5,9$ and $\operatorname{dim} \phi=5$;

(d) $\ell=3, g \in 3 c, 3 d, 4 b$ or $|g|=5,9$ and $\operatorname{dim} \phi=5$;

(e) $\ell \neq 3, g \in 3 c$ or $|g|=5,9$ and $\operatorname{dim} \phi=6$.

- $G=L .2$ and

(f) $\ell=3, g \in 4 d$ or $|g|=8$ and $\operatorname{dim} \phi=4$;

(g) $\ell=3,|g|=4,8$ and $\operatorname{dim} \phi=5$;

(h) $\ell \neq 3,|g|=4,8$ and $\operatorname{dim} \phi=6$.

Clearly, if we view $L$ as $\operatorname{PSU}_{4}(2)$ we must add the extra condition $\ell \neq 2$ to items (a), (e) and (h), whereas if we view $L$ as $\mathrm{PSp}_{4}(3)$ we must ignore items (b), (d), (f) and $(\mathrm{g})$. Also observe that in item (c) the assumption $\ell \neq 2$ is due to the fact that $\mathrm{PSp}_{4}(3)$ does not have 2-modular irreducible representations of degree 5 .

6.7. $L=\mathrm{PSU}_{4}(3)$. In this case, $\operatorname{dim} \phi \geq 6$. Observing that if $g \notin L$, then $g$ is a 2-element, and using the GAP package, we get the following evidences for the values of $\alpha(g)$ and the almost cyclicity condition on $\phi(g)$ :

\begin{tabular}{c|c|c|c||c|c|c|c}
$|g|$ & & $\alpha(g)$ & $\operatorname{dim} \phi \leq$ & $|g|$ & & $\alpha(g)$ & $\operatorname{dim} \phi \leq$ \\
\hline 3 & $g \in L$ & $\leq 4$ & $4(3-1)=8$ & 7 & $g \in L$ & $=2$ & $2(7-1)=12$ \\
4 & $g \in L$ & $=2$ & $2(4-1)=6$ & 8 & $g \in L$ & $=2$ & $2(8-1)=14$ \\
4 & $g \notin L$ & $\leq 4$ & $4(4-1)=12$ & 8 & $g \notin L$ & $=2$ & $2(8-1)=14$ \\
5 & $g \in L$ & $=2$ & $2(5-1)=8$ & 9 & $g \in L$ & $=2$ & $2(9-1)=16$
\end{tabular}

It follows that we only have to examine the representations $\phi$ such that $6 \leq$ $\operatorname{dim} \phi \leq 16$. It turns out that if either $\ell=0$ or $(p, \ell)=1$, then $\phi(g)$ is almost cyclic if and only if $\operatorname{dim} \phi=6$ and

- $G=L, g \in 3 b$ or $|g|=5,7,8,9$;

- $G=L .2_{2}, g \in 4 d$ or $|g|=8$ and $\ell \neq 2,3$.

Next, suppose that $\ell=p$. Then, for $\ell=2,5,7$, we are left to examine the following possibilities:

(1) $\ell=2, G \in\left\{L, L .2_{2}\right\},|g|=4,8$ and $\operatorname{dim} \phi=6$;

(2) $\ell=2, G=L .2_{1},|g|=4,8$ and $\operatorname{dim} \phi=12$;

(3) $\ell=5, G=L,|g|=5$ and $\operatorname{dim} \phi=6$;

(4) $\ell=7, G=L,|g|=7$ and $\operatorname{dim} \phi=6$.

Considering (1), we see that if $|g|=8$, then, since $\operatorname{dim} \phi=6, \phi(g)$ must have a single non-trivial block of size $\geq 5$. Thus $\phi(g)$ is almost cyclic. So, assume that $|g|=4$. Assume first that $g \in L$. There are two classes of elements of order 4 in $L$, namely $4 a, 4 b$. If $g \in 4 a$, w.l.o.g we may assume that $g$ lies in a maximal subgroup $M$ of $L$ isomorphic to $\mathrm{PSU}_{3}(3)$. We find that $\phi$ restricted to $M$ remains irreducible and $\phi(g)$ is the sum of two Jordan blocks of size 3. So $\phi(g)$ is not almost cyclic. If $g \in 4 b$, w.l.o.g we may assume that $g$ lies in a maximal $\operatorname{subgroup~} N$ of $L$ isomorphic to $A_{7}$. We find that $\phi$ restricted to $N$ remains irreducible and $\phi(g)$ is the sum of two Jordan blocks of size 4 and 2 . So again $\phi(g)$ is not almost cyclic.

Next, assume that $g \notin L$ and $\langle L, g\rangle=L .2_{2}$. There are two classes of such elements, namely $4 c, 4 d$. If $g \in 4 c$, w.l.o.g we may assume that $g$ lies in a maximal subgroup $P$ of $L$ isomorphic to $\operatorname{PSU}_{3}(3) .2$. We find that $\phi$ restricted to $P$ remains irreducible and $\phi(g)$ is the sum of two Jordan blocks of size 3. So $\phi(g)$ is not almost cyclic. If $g \in 4 d$, w.l.o.g we may assume that $g$ lies in a maximal subgroup $Q$ of $L$ 
isomorphic to $S_{7}$. We find that $\phi$ restricted to $Q$ remains irreducible and $\phi(g)$ is the sum of two Jordan blocks of size 1 and one of size 4 . So, $\phi(g)$ is almost cyclic.

The instances in (2) can be ruled out, as a 2-modular 12-dimensional representation of $G$ when restricted to $L$ splits into two 6-dimensional components intertwined by $g$. Now, $\phi(g)$ is almost cyclic if and only if $g^{2}$ is cyclic on each component, but this cannot be by [4, Proposition 2.14].

Finally, observe that the Sylow 5-subgroups as well as the Sylow 7-subgroups are cyclic. It follows that in instances (3) and (4), by [6, Lemma 2.13 and Corollary 2.14], $\phi(g)$ is almost cyclic (in fact, cyclic in instance (4)).

We conclude that, for any value of $\ell, \phi(g)$ is almost cyclic if and only if $\operatorname{dim} \phi=6$ and one of the following occurs:

- $G=L$ and either $g \in 3 b$ or $|g|=5,7,8,9$;

- $G=L .2_{2}$ and either $g \in 4 d$ or $|g|=8$.

Remark. Note that in the following cases $\left.\phi\right|_{L}$ is an irreducible Weil representation of $L$, and $g$ is a semisimple element. Thus, they have been already described in 6 .

(1) $G=\mathrm{PSL}_{3}(2), \operatorname{dim} \phi=5, \ell=7$ and $|g|=7$;

(2) $G=\mathrm{PSL}_{3}(2), \operatorname{dim} \phi=7$ and $|g|=7$

(3) $G=\operatorname{PSU}_{3}(3), \operatorname{dim} \phi=6$ and $|g|=7,8$;

(4) $G=\mathrm{PSU}_{3}(3), \operatorname{dim} \phi=7, \ell \neq 2$ and $|g|=7,8$;

(5) $G=\mathrm{PSU}_{4}(2), \operatorname{dim} \phi=5$ and $g \in 3 d$ or $|g|=5,9$;

(6) $G=\mathrm{PSU}_{4}(2), \operatorname{dim} \phi=5, \ell=3$ and $g \in 3 c$;

(7) $G=\mathrm{PSU}_{4}(2), \operatorname{dim} \phi=6, \ell \neq 3$ and $g \in 3 c$ or $|g|=5,9$;

(8) $G=\mathrm{PSp}_{4}(3), \operatorname{dim} \phi=4$ and $|g|=4,5$;

(9) $G=\mathrm{PSp}_{4}(3), \operatorname{dim} \phi=5, \ell \neq 2$ and $g \in 4 b$ or $|g|=5$.

7. The GROUPS $\operatorname{Sp}_{2 n}(q), n>1, q$ EVEN; $\Omega_{2 n+1}(q), n>2, q$ ODD; $\mathrm{P} \Omega_{2 n}^{ \pm}(q)$, $n>3$

As shown in [12, Theorem 2.16], if $L$ is one of the simple groups $\operatorname{Sp}_{2 n}(q)(n \geq 2$ and $q$ even), $\Omega_{2 n+1}(q)\left(n \geq 3\right.$ and $q$ odd) or $\mathrm{P}_{2 n}^{ \pm}(q)(n \geq 4)$, then $|g| \leq \frac{q^{n+1}}{q-1}$ for all $g \in$ Aut $L$.

Lemma 7.1. Let $L=\operatorname{Sp}_{2 n}(q)$, where $n \geq 2, q$ is even and $(n, q) \neq(4,2)$. Let $g \in$ Aut $L$, where $g^{2} \neq 1$, and let $G=\langle L, g\rangle \subseteq$ Aut $L$. Let $\phi$ be a cross-characteristic projective irreducible representation $\phi$ of $G$ that is non-trivial on $L$. Then $\phi(g)$ is almost cyclic if and only if one of the following occurs:

(1) $G=\operatorname{Sp}_{4}(4),|g|=17$ and $\operatorname{dim} \phi=18$.

(2) $G=\operatorname{Sp}_{6}(2)$ and

(a) $\operatorname{dim} \phi=7$ and either $g \in 3 a, 4 c$, or $|g|=5,7,8,9$;

(b) $\operatorname{dim} \phi=8$ and $|g|=7,8,9$.

Proof. First, recall that $\operatorname{dim} \phi \geq \frac{\left(q^{n}-1\right)\left(q^{n}-q\right)}{2(q+1)}$ and $\alpha(g) \leq 2 n+1$ for any $g \in$ Aut $L$. Suppose that $\phi(g)$ is almost cyclic. By Lemma 2.4 this implies that $\frac{\left(q^{n}-1\right)\left(q^{n}-q\right)}{2(q+1)} \leq$ $(2 n+1)(|g|-1) \leq(2 n+1) \frac{q^{n+1}-q+1}{q-1}$. The above inequality holds if and only if $(2 n, q) \in\{(4,4),(4,8),(6,2),(6,4),(8,2),(10,2),(12,2),(14,2)\}$. Thus, almost cyclicity can only occur for these values of the pair $(2 n, q)$. However, it is to check that, unless $(2 n, q)=(4,4),(6,2), \phi(g)$ cannot be almost cyclic, by Lemma 2.4 . 
If $L=\operatorname{Sp}_{4}(4)$, then $\operatorname{dim} \phi \geq 18$ and $|g| \in\{3,4,5,8,17\}$. If $|g|=4,8,17$, then $\alpha(g)=2$; if $|g|=3,5$, then $\alpha(g) \leq 3$. If $|g|<17$, then $18>\alpha(g)|g|$, and hence $\phi(g)$ is not almost cyclic. If $|g|=17$, then $g \in L$, and using the GAP package, we see that $\phi(g)$ is almost cyclic if and only if $\operatorname{dim} \phi=18$. Whence item (1) of the statement.

If $L=\operatorname{Sp}_{6}(2)$, then $|g| \in\{3,4,5,7,8,9\}$ and $\operatorname{dim} \phi \geq 7$. If $|g|=3$, then $\alpha(g) \leq 4$; if $|g|=4$, then $\alpha(g) \leq 3$; finally, if $|g| \geq 5$, then $\alpha(g)=2$. Thus $\phi(g)$ is not almost cyclic, unless possibly when $\operatorname{dim} \phi \leq 16$. Using the GAP package, we see that $\phi(g)$ is almost cyclic if and only if one of the instances listed in item (2) of the statement occurs.

Remark 7.2. The group $\mathrm{Sp}_{4}(2)$ is not simple. However, the commutator subgroup $L=\operatorname{Sp}_{2}(2)^{\prime}$ is simple and isomorphic to $\mathrm{PSL}_{2}(9)$. Using the package GAP and assuming that $g$ is not involution, we find that $\phi(g)$ is almost cyclic if and only if $G=L$ and the following holds:

(1) $\operatorname{dim} \phi=3,4,5, \ell \neq 2,3$ and $|g|=3,4,5$;

(2) $\operatorname{dim} \phi=6, \ell \neq 2$ and $|g|=5$;

(3) $\operatorname{dim} \phi=3,4, \ell=2$ and $|g|=3,4,5$;

(4) $\operatorname{dim} \phi=2,3,4, \ell=3$ and $|g|=3,4,5$.

Lemma 7.3. Let $L=\Omega_{2 n+1}(q)$, where $n \geq 3$ and $q$ is odd, or $L=\mathrm{P} \Omega_{2 n}^{-}(q)$, where $n \geq 4$. Let $g \in$ Aut $L$, where $g^{2} \neq 1$, and let $G=\langle L, g\rangle \subseteq$ Aut $L$. Then $\phi(g)$ is not almost cyclic in any cross-characteristic projective irreducible representation $\phi$ of $G$ that is non-trivial on $L$.

Proof. Suppose that $L=\Omega_{2 n+1}(q)$, and assume that $\phi(g)$ is almost cyclic. By Lemma 2.8. if $q=3$ and $n \geq 4$, then $\operatorname{dim} \phi \geq \frac{\left(3^{n}-1\right)\left(3^{n}-3\right)}{8}$, whence the bound

$$
\frac{\left(3^{n}-1\right)\left(3^{n}-3\right)}{8} \leq(2 n+1) \frac{3^{n+1}-2}{2},
$$

which holds if and only if $n=4$. If $q>3$, then, again by Lemma 2.8, $\operatorname{dim} \phi \geq$ $\frac{q^{2 n}-1}{q^{2}-1}-2$, whence the bound

$$
\frac{q^{2 n}-2 q^{2}+1}{q^{2}-1} \leq(2 n+1) \frac{q^{n+1}-q+1}{q-1},
$$

which holds if and only if $n=3$ and $q=5,7$. By the above, we are left to examine the cases where $(n, q) \in\{(3,3),(3,5),(3,7),(4,3)\}$. In each of these cases, it is routine to check that $\phi(g)$ is not almost cyclic, by Lemma 2.4.

Now, suppose that $L=\mathrm{P} \Omega_{2 n}^{-}(q)$ and assume that $\phi(g)$ is almost cyclic. By Lemma 2.8, (8), $\operatorname{dim} \phi \geq \frac{\left(q^{n}+1\right)\left(q^{n-1}-q\right)}{q^{2}-1}-1$, provided $(n, q) \neq(4,2),(4,4),(5,2)$, $(5,3)$. In the first case, the following bound must be met:

$$
\frac{\left(q^{n}+1\right)\left(q^{n-1}-q\right)-\left(q^{2}-1\right)}{q^{2}-1} \leq 2 n \frac{q^{n+1}-q+1}{q-1} .
$$

However, this only holds if either $n=4$ and $q \leq 8$ or $n=6,7$ and $q=2$. Thus, we are left to examine the following possible exceptional cases: $(n, q)=(4,2)$, $(4,3),(4,4),(4,5),(4,7),(5,2),(5,3),(6,2),(7,2)$. In each individual case, from the available data we get $\operatorname{dim} \phi>\alpha(g)(|g|-1)$, a contradiction. 
Lemma 7.4. Let $L=\mathrm{P} \Omega_{2 n}^{+}(q)$, where $n \geq 4$. Let $g \in$ Aut $L$, where $g^{2} \neq 1$, and set $G=\langle L, g\rangle \subseteq$ Aut $L$. Let $\phi$ be a cross-characteristic projective irreducible representation of $G$ non-trivial on $L$. Then $\phi(g)$ is almost cyclic if and only if $L=\Omega_{8}^{+}(2), \operatorname{dim} \phi=8$ and one of the following occurs:

- $G=L$ and either $g \in 3 a, 5 a, 8 b$ or $|g|=7,9$;

- $G=\mathrm{SO}_{8}^{+}(2)$ and either $g \in 4$ for $|g|=8$.

Proof. Suppose that $\phi(g)$ is almost cyclic. If $q \leq 3$ and $(n, q) \neq(4,2)$, then $\operatorname{dim} \phi \geq$ $\frac{\left(q^{n}-1\right)\left(q^{n-1}-1\right)}{q^{2}-1}$. It follows that the bound

$$
\frac{\left(q^{n}-1\right)\left(q^{n-1}-1\right)}{q^{2}-1} \leq 2 n \frac{q^{n+1}-q+1}{q-1}
$$

must be met. This happens if and only if either $n \leq 5$ or $q=2$ and $n=6,7$. If $q>3$, then $\operatorname{dim} \phi \geq \frac{\left(q^{n}-1\right)\left(q^{n-1}+q\right)}{q^{2}-1}-2$, and so the bound to be met is

$$
\frac{\left(q^{n}-1\right)\left(q^{n-1}+q\right)-2\left(q^{2}-1\right)}{q^{2}-1} \leq 2 n \frac{q^{n+1}-q+1}{q-1} .
$$

This holds if and only if $n=4$ and $q=4,5,7,8$, and in each case knowledge of $\alpha(g)$ and $|g|$ yields a contradiction by Lemma 2.4 .

Finally, let us deal with the case when $L=\Omega_{8}^{+}(2)$. Here $|g| \in\{3,4,5,7,8,9\}$ and $\operatorname{dim} \phi \geq 8$ (see Lemma 2.8). (Indeed, $L$ does have a (unique) projective representation of degree 8 for any characteristic $\ell$, and this is not an ordinary representation of $L)$.

Suppose first that $g \in \mathrm{SO}_{8}^{+}(2)$. If $|g|=3$, then $\alpha(g) \leq 4$; if $|g|=4$, then $\alpha(g) \leq 3$; if $|g| \geq 5$, then $\alpha(g)=2$. Since either $\operatorname{dim} \phi=8$ or $\operatorname{dim} \phi \geq 28$, applying the usual bound we are only left to consider the case when $\operatorname{dim} \phi=8$.

Let $G=L$. If $\ell \neq p$, then using the GAP package we find that $\phi(g)$ is almost cyclic if and only if $g$ is one of elements described in the statement.

Now, suppose that $\ell=p=7$. Since the Sylow 7-subgroups are cyclic, for $\ell=7$ we can refer to [6. Lemma 2.13 and Corollary 2.14], and we find that $\phi(g)$ is almost cyclic.

Next, suppose that $\ell=p=5$. We observe that $L=\Omega_{8}^{+}(2)$ contains three classes of elements of order 5 and three classes of maximal subgroups isomorphic to $\mathrm{Sp}_{6}(2)$. Each of these symplectic groups contains elements from a single conjugacy class of elements $g \in L$ of order 5 and each element of order 5 of $L$ is contained in a symplectic group. (Note that the same holds for elements of order 9 , considering the three classes $9 a, 9 b, 9 c$ of elements of order 9 and the three classes of maximal subgroups isomorphic to $A_{9}$.)

Now, considering the group 2.L, we see that the elements of the class $5 a$ belong to a maximal subgroup $M$ of type $2 \times \operatorname{Sp}_{6}(2)$ (a single class). The restriction of $\phi$ to $M$ decomposes as the sum of a representation of degree 1 and a representation $\psi$ of degree 7. Since $\psi(g)$ is almost cyclic (see Lemma 7.1), it follows that $\phi(g)$ is almost cyclic.

On the contrary, the elements of the classes $5 b$ and $5 c$ belong to a maximal subgroup $N$ of type $2 . \mathrm{Sp}_{6}(2)$ (non-split central extensions: there are two classes of such subgroups). The representation $\phi$ of degree 8 of $2 . L$ restricts irreducibly to $N$ and, working in $N$, we see that $\phi(g)$ is not almost cyclic.

Finally, suppose that $\ell=p=3$. The group $L$ has five classes $3 a, 3 b, 3 c, 3 d, 3 e$ of elements of order 3 , and three classes $9 a, 9 b, 9 c$ of elements of order 9 . Each 
of the classes $3 a, 3 b, 3 c$, and similarly each of the classes $9 a, 9 b, 9 c$ is contained in a unique maximal subgroup isomorphic to $\operatorname{Sp}_{6}(2)$, and conversely each of these symplectic groups contains elements from a single conjugacy class of elements of order 3 and 9 of $L$, respectively.

On the other hand, if $g$ belongs to one of the classes $3 d, 3 e$, then $\alpha(g)=3$. This yields a contradiction, by Lemma 2.4

Next, considering the group 2.L, we see that the elements of classes $3 b, 3 c, 9 b, 9 c$ belong to a maximal subgroup $M$ of type $2 . \mathrm{Sp}_{6}(2)$ (there are two classes of such subgroups). The representation $\phi$ of degree 8 of $2 . L$ restricts irreducibly to $M$ and, working in $M$, we obtain that $\phi(g)$ is almost cyclic if and only if $g$ has order 9 .

On the other hand, the elements $g$ of the classes $3 a, 9 a$ belong to a maximal subgroup $N$ of type $2 \times \operatorname{Sp}_{6}(2)$ (a single class). The restriction of $\phi$ to such subgroups decomposes as the sum of a representation of degree 1 and a representation $\psi$ of degree 7 . Since $\psi(g)$ is almost cyclic, $\phi(g)$ is also almost cyclic.

Next, let $G=\langle L, g\rangle=\mathrm{SO}_{8}^{+}(2)$. Then $p=2$, and using the GAP package one can check that $\phi(g)$ is almost cyclic if and only if either $|g| \in 4 f$ or $|g|=8$, as claimed.

Finally, suppose that $g \notin \mathrm{SO}_{8}^{+}(2)$, and hence $|g|=3,9$. Then $\alpha(g) \leq 8$; moreover, either $\operatorname{dim} \phi \leq 50$ or $\operatorname{dim} \phi \geq 105$ (see [1] and 20]). If $\ell \neq 3$, then use of the GAP package shows that $\phi(g)$ is not almost cyclic. If $\ell=3$, we need to consider representations of degree 28 or 48 . Applying the usual bound, we can easily see that $\phi(g)$ is not almost cyclic if $|g|=3$. Now, let $g \notin L$ be an element of order 9 belonging so a class $C$. Then the power maps in [1] show that $g^{3}$ belongs to the class $3 d$ of $L$, and therefore every element $x \in 3 d$ is the cube of some $y \in C$. We find that we can choose three elements in $3 d$, say $x_{1}, x_{2}, x_{3}$, which generate $L$. Let $g_{1}, g_{2}, g_{3}$ three elements of $C$ such that $x_{i}=g_{i}^{3}$ for $i=1,2,3$. Thus, the group $H=\left\langle g_{1}, g_{2}, g_{3}\right\rangle$ contains $\left\langle g_{1}^{3}, g_{2}^{3}, g_{3}^{3}\right\rangle=L$, whence $H=L .3$.

The above implies that $\alpha(g) \leq 3$ for all $g \in C$. Thus, by Lemma 2.4, $\phi(g)$ cannot be almost cyclic.

\section{The EXCeptional GROUPs of LiE Type}

In this section we deal with the groups Aut $L$, where $L$ is a finite simple exceptional group of Lie type. The notation for the groups $L$ is standard. Note however that our notation for $q$ in the case of twisted groups is such that ${ }^{2} E_{6}(q) \subset E_{6}\left(q^{2}\right)$, ${ }^{3} D_{4}(q) \subset D_{4}\left(q^{3}\right),{ }^{2} B_{2}(q) \subset B_{2}(q),{ }^{2} F_{4}(q) \subset F_{4}(q)$ and ${ }^{2} G_{2}(q) \subset G_{2}(q)$. We also recall that the structure of Aut $L$ can be found in [23, Table 5.1B]. For a thorough and detailed reference, however, see [10, Section 2.5 (Theorem 2.5.12)].

The maximal order of an element in Aut $L$ can be easily deduced from [12, 21]: these data are summarised in Table 2. Lower bounds for the dimension of a nontrivial cross-characteristic projective representation of $L$ are found in [19, Table 1, p. 14]. Finally, we recall that, for any non-trivial element $g \in$ Aut $L$, the minimal number $\alpha(g)$ of $L$-conjugates of $g$ sufficient to generate $\langle g, L\rangle$ is provided by Proposition 2.2(2).

Lemma 8.1. Let $L$ be a simple exceptional group of Lie type. Let $1 \neq g \in$ Aut $L$ be a p-element and let $G=\langle g, L\rangle$. Then $g$ is almost cyclic in some cross-characteristic projective irreducible representation $\phi$ of $G$ that is non-trivial on $L$ if and only if one if one of the cases listed in Table 3 below occurs. 


\begin{tabular}{ccc}
$L$ & Conditions & $|g| \leq$ \\
\hline${ }^{2} B_{2}\left(2^{2 e+1}\right)$ & $e \geq 1$ & $(2 e+1)\left(2^{2 e+1}+2^{e+1}+1\right)$ \\
$G_{2}(q)$ & $q=r^{e}, r \neq 3$ & $e\left(q^{2}+q+1\right)$ \\
$G_{2}(q)$ & $q=3^{e}$ & $2 e\left(q^{2}+q+1\right)$ \\
${ }^{2} G_{2}\left(3^{2 e+1}\right)$ & $e \geq 1$ & $(2 e+1)\left(3^{2 e+1}+3^{e+1}+1\right)$ \\
${ }^{3} D_{4}(q)$ & $q=r^{e}$ & $3 e\left(q^{3}-1\right)(q+1)$ \\
$F_{4}(q)$ & & $q\left(q^{3}-1\right)(q+1)$ \\
${ }^{2} F_{4}\left(2^{2 e+1}\right)$ & $e \geq 1$ & $(2 e+1)\left(2^{4 e+2}+2^{3 e+2}+2^{2 e+1}+2^{e+1}+1\right)$ \\
$E_{6}(q)$ & & $q^{2}\left(q^{3}+1\right)\left(q^{2}+q+1\right)$ \\
${ }^{2} E_{6}(q)$ & & $q(q+1)\left(q^{2}+1\right)\left(q^{3}-1\right)$ \\
$E_{7}(q)$ & & $q(q+1)\left(q^{2}+1\right)\left(q^{4}+1\right)$ \\
$E_{8}(q)$ & & $q(q+1)\left(q^{2}+q+1\right)\left(q^{5}-1\right)$
\end{tabular}

TABle 2. Maximal order of an element in Aut $L$.

Proof. Suppose that $\phi(g)$ is almost cyclic.

If $L={ }^{2} B_{2}\left(2^{2 e+1}\right)$, where $e \geq 2$, then $\alpha(g) \leq 5$. By Lemma 2.4 and Tables 2 and [19, Table 1, p. 14], the bound $2^{e}\left(2^{2 e+1}-1\right) \leq 5\left((2 e+1)\left(2^{2 e+1}+2^{e+1}+1\right)-1\right)$ must be met. However, the bound only holds for $e \leq 6$. So, we may assume $2 \leq e \leq 6$. If $g \in L$, then $|g| \leq 2^{2 e+1}+2^{e+1}+1$, and hence we can refine the bound to $2^{e}\left(2^{2 e+1}-1\right) \leq 5\left(2^{2 e+1}+2^{e+1}\right)$. However, this only holds for $e=2$. This means that for $e=3,4,5,6$, it suffices to examine elements $g \notin L$. But, for these values of $e,|g| \leq 2 e+1$, which yields $2^{e}\left(2^{2 e+1}-1\right)>10 e$, a contradiction.

If $L={ }^{2} B_{2}\left(2^{3}\right)$, then $|g| \in\{2,3,4,5,7,13\}$ and $\operatorname{dim} \phi \geq 8$. If $|g|=2, \alpha(g)=3$, whereas, if $|g|>2, \alpha(g)=2$. We readily get a contradiction if $|g|=2,3,4$. So, we may assume $|g| \geq 5$, which implies $g \in L$. Inspection of the Brauer characters shows that we are left to consider the following cases: (i) $|g|=5,7,13, \ell=5$ and $\operatorname{dim} \phi=8$; (ii) $|g|=13, \ell \neq 2$ and $\operatorname{dim} \phi=14$; (iii) $|g|=13, \ell=13$ and $\operatorname{dim} \phi=16,24$. Using the GAP package we obtain item (1) of the statement. If $L={ }^{2} B_{2}\left(2^{5}\right)$, then $|g| \in\{2,4,5,25,31,41\}$ and $\operatorname{dim} \phi \geq 124$. Since $\alpha(g) \leq 3$, we get a contradiction.

Next, suppose that $L=G_{2}(q)$. If $q \geq 5$, then a detailed check shows that we get a contradiction, by Lemma 2.4.

Next, let $L=G_{2}(3)$. Then $|g| \in\{2,3,4,7,8,9,13\}$ and either $\operatorname{dim} \phi=14$ or $\operatorname{dim} \phi \geq 27$. If $|g| \leq 3$, then $\alpha(g) \leq 4$, otherwise $\alpha(g)=2$. Thus we readily get a contradiction, unless $|g|=8,9,13$ and $\operatorname{dim} \phi=14$. Using the GAP package, we find that $\phi(g)$ is almost cyclic only for $|g|=13$.

Finally, let $L=G_{2}(4)$. Then $|g| \in\{2,3,4,5,7,8,13,16\}$ and either $\operatorname{dim} \phi=12$ or $\operatorname{dim} \phi \geq 64$. If $|g| \geq 4$, then $\alpha(g)=2$, whereas $\alpha(g) \leq 4$ if $|g| \leq 3$. We readily get a contradiction, unless $\operatorname{dim} \phi=12$ and $|g| \geq 7$. Using the GAP package, we find that $\phi(g)$ is almost cyclic if and only if $|g|=13,16$.

Now, suppose that $L={ }^{3} D_{4}(q)$, where $q=r^{e}$. Ruling out this group requires a rather delicate analysis, which we need to develop in detail. Here $\alpha(g) \leq 7$ and we have to consider the bound $r^{3 e}\left(r^{2 e}-1\right) \leq 7\left(3 e\left(r^{4 e}+r^{3 e}-r^{e}-1\right)-1\right)$, which only holds when $q$ has one of the following values: $q=2^{e}$, where $e \leq 7 ; q=3^{e}$, where $e \leq 4 ; q=5^{e}$, where $e \leq 2 ; q=7,11,13,17,19$. 
Suppose first that $g$ is semisimple. From the description of the maximal tori of $L$ given in [22, we can extract the following table, which gives, for each value of $q$, an upper bound $s(q)$ for the maximal order of a semisimple $p$-element of Aut $L$ :

\begin{tabular}{c|c||c|c||c|c||c|c||c|c||c|c}
$q$ & $s(q)$ & $q$ & $s(q)$ & $q$ & $s(q)$ & $q$ & $s(q)$ & $q$ & $s(q)$ & $q$ & $s(q)$ \\
\hline 2 & 13 & 3 & 73 & 4 & 241 & 5 & 601 & 7 & 181 & 8 & 243 \\
9 & 6481 & 11 & 1117 & 13 & 28393 & 16 & 673 & 17 & 83233 & 19 & 769 \\
25 & 390001 & 27 & 530713 & 32 & 1321 & 64 & 38737 & 81 & 6481 & 128 & 14449
\end{tabular}

It is easy to verify that for $q \geq 7$ we get a contradiction, by Lemma 2.4 .

Next, suppose that $g$ is unipotent. Then $\eta_{p}(L)=p$ if $p \geq 3$, while $\eta_{2}(L)=8$ and $\eta_{3}(L)=9$ (see [28]). Thus we readily get a contradiction for $q \geq 3$.

Let $L={ }^{3} D_{4}(2)$. Then $|g| \in\{2,3,4,7,8,9,13\}$ and $\operatorname{dim} \phi \geq 25$. If $|g| \geq 4$, then $\alpha(g)=2$, whereas, if $|g|=2,3$, then $\alpha(g) \leq 4$. In both cases, we get a contradiction.

At this stage, we are left to examine the cases $q=3,4,5$ and $g$ semisimple.

Let $L={ }^{3} D_{4}(3)$. Then $|g| \in\{2,4,7,8,13,73\}$. Since $\operatorname{dim} \phi \geq 216$, we get a contradiction unless $|g|=73$. In this case, using the GAP package, we find that $\alpha(g)=2$, which yields a contradiction.

Let $L={ }^{3} D_{4}(4)$. Then $|g| \in\{3,5,7,9,13,27,241\}$. Since $\operatorname{dim} \phi \geq 960$, we get a contradiction unless $|g|=241$. Finally, let $L={ }^{3} D_{4}(5)$. Then $|g| \in$ $\{2,3,4,7,8,9,31,601\}$. Since $\operatorname{dim} \phi \geq 3000$, we get a contradiction unless $|g|=601$.

Notice that in the instances $|g|=241$ for $L={ }^{3} D_{4}(4)$, and $|g|=601$ for $L=$ ${ }^{3} D_{4}(5)$, the exceptional element $g$ belongs to $L$ and is a generator of the cyclic torus $T_{5}$ (in the notation of [22]). Since $g$ is regular semisimple, by Lemma 2.7 we have $\alpha(g) \leq 3$, whence a contradiction.

Finally, we list in Table 3 the exact occurrences of almost cyclic elements for the simple group ${ }^{2} F_{4}(2)^{\prime}$, the so-called Tits group, as well as for the simple groups $G=G_{2}(2)^{\prime}$ and $G={ }^{2} G_{2}(3)^{\prime}$. These results are based, as usual, on Lemma 2.4 and on direct computations with GAP. All the remaining exceptional groups are ruled out using Lemma 2.4. The relevant computations are left to the reader.

\begin{tabular}{c|c|c|c||c|c|c|c}
$G$ & $\ell$ & $\operatorname{dim} \phi$ & $|g|$ & $G$ & $\ell$ & $\operatorname{dim} \phi$ & $|g|$ \\
\hline${ }^{2} B_{2}\left(2^{3}\right)$ & all & 14 & 13 & ${ }^{2} B_{2}\left(2^{3}\right)$ & 5 & 8 & 7,13 \\
$G_{2}(2)^{\prime}$ & all & 6,7 & 7,8 & $G_{2}(2)^{\prime}$ & 3 & 3 & all \\
$G_{2}(2)$ & all & 6,7 & 8 & $G_{2}(3)$ & all & 14 & 13 \\
$G_{2}(4)$ & all & 12 & 13 & $G_{2}(4) .2$ & all & 12 & 16 \\
${ }^{2} G_{2}(3)^{\prime}$ & $\neq 2$ & 7,8 & 7,9 & ${ }^{2} G_{2}(3)^{\prime}$ & $\neq 2,7$ & 9 & 9 \\
${ }^{2} G_{2}(3)^{\prime}$ & 2 & 2 & all & ${ }^{2} G_{2}(3)^{\prime}$ & 2 & 4 & $3,7,9$ \\
${ }^{2} G_{2}(3)^{\prime}$ & 2 & 8 & 7,9 & ${ }^{2} G_{2}(3)$ & $\neq 2$ & 7,8 & 9 \\
${ }^{2} G_{2}(3)$ & 2 & 6,8 & 9 & & & &
\end{tabular}

TABLE 3. Occurrences of almost cyclic elements in exceptional groups of Lie type. 


\section{Proof of the Main Theorem}

The following theorem answers with full details the question raised in the present paper, thus also proving the Main Theorem quoted in the Introduction.

Theorem 9.1. Let $L$ be a finite simple group of Lie type, let $g \in$ Aut $L$ be a p-element for some prime $p$, and let $G=\langle L, g\rangle$. Let $\phi$ be an irreducible projective representation of $G$ over an algebraically closed field $\mathbb{F}$ of characteristic $\ell$, different from the defining characteristic of $L$. Suppose that $\phi$ is non-trivial on $L$; furthermore, assume that $g^{2} \neq 1, L \neq \operatorname{PSL}_{2}(q)$ and $\phi(g)$ is almost cyclic. Then, either $\phi_{\mid L}$ is an irreducible Weil representation of $L$, where $L \in\left\{\operatorname{PSL}_{n}(q)(n \geq\right.$ $3), \operatorname{PSU}_{n}(q)(n \geq 3), \operatorname{PSp}_{2 n}(q)(n \geq 2, q$ odd $\left.)\right\}$ and $g$ is semisimple, or $L$ is of exceptional type and $(\operatorname{dim} \phi, \ell,|g|)$ are given in Table 3 , or one of the following occurs:

(1) $L=\operatorname{PSL}_{3}(2), \operatorname{dim} \phi=2, \ell=7$ and either $|g|=3,4,7$ or $g \notin L$ and $|g|=8$;

(2) $L=\mathrm{PSL}_{3}(2), \operatorname{dim} \phi=3,4$ and and either $|g|=3,4,7$ or $g \notin L$ and $|g|=8$;

(3) $L=\operatorname{PSL}_{3}(2), \operatorname{dim} \phi=5, \ell=7$ and either $|g|=4$ or $g \notin L$ and $|g|=8$;

(4) $G=\operatorname{PSL}_{3}(2), \operatorname{dim} \phi=6, \ell=7$ and $|g|=7$;

(5) $G=\operatorname{PSL}_{3}(2) .2, \operatorname{dim} \phi=6,7$, and $|g|=8$, with $g \notin L$;

(6) $L=\operatorname{PSL}_{3}(2)$, $\operatorname{dim} \phi=8$ and either $\ell \neq 3,7$ and $|g|=7$, or $\ell \neq 7, g \notin L$ and $|g|=8$;

(7) $G=\mathrm{PSL}_{3}(4), \operatorname{dim} \phi=4, \ell=3$ and $|g|=3,4,5,7$;

(8) $G=\mathrm{PSL}_{3}(4), \operatorname{dim} \phi=6$ and $|g|=5,7$;

(9) $G=\operatorname{PSL}_{3}(4)$, $\operatorname{dim} \phi=8$ and $|g|=7$;

(10) $G=\operatorname{PSL}_{3}(4) .2_{1}$, $\operatorname{dim} \phi=6$ and $|g|=8$;

(11) $G=\operatorname{PSL}_{3}(4) .2_{2}$, $\operatorname{dim} \phi=4,6, \ell=3$ and $|g|=8$;

(12) $G=\operatorname{PSL}_{3}(4) .2_{3}, \operatorname{dim} \phi=6, \ell=3$ and $|g|=8$;

(13) $G=\operatorname{PSL}_{3}(4) .2_{3}$, $\operatorname{dim} \phi=8$ and $|g|=8$;

(14) $G=\operatorname{PSL}_{3}(4) .2_{3}, \operatorname{dim} \phi=10, \ell=3$ and $g \in 8 a$;

(15) $G=\mathrm{PSL}_{4}(2), \operatorname{dim} \phi=7$ and either $g \in 3 a$ or $|g|=5,7$;

(16) $G=\mathrm{PSL}_{4}(2), \operatorname{dim} \phi=8$ and $|g|=7$;

(17) $G=\mathrm{PSL}_{4}(2) .2, \operatorname{dim} \phi=7$ and $|g|=4,8$;

(18) $G=\mathrm{PSL}_{4}(2) .2, \operatorname{dim} \phi=8$ and $|g|=8$;

(19) $G=\operatorname{PSU}_{3}(3) .2$, $\operatorname{dim} \phi=6$ and $|g|=8$;

(20) $G=\operatorname{PSU}_{3}(3) .2, \operatorname{dim} \phi=7, \ell \neq 2$ and $|g|=8$;

(21) $G=\operatorname{PSU}_{3}(4), \operatorname{dim} \phi=12$ and $|g|=16$;

(22) $G=\mathrm{PSU}_{4}(2), \operatorname{dim} \phi=4, \ell \neq 3$ and $g \in 3 a, 3 b, 3 d$ or $|g|=4,5,9$;

(23) $G=\mathrm{PSU}_{4}(2), \operatorname{dim} \phi=4, \ell=3$ and $g \in 3 a, 3 b, 4 a$ or $|g|=5,9$;

(24) $G=\mathrm{PSU}_{4}(2)$, $\operatorname{dim} \phi=5$ and $g \in 4 b$;

(25) $G=\mathrm{PSU}_{4}(2) .2, \operatorname{dim} \phi=4, \ell=3$ and $g \in 4 d$ or $|g|=8$;

(26) $G=\mathrm{PSU}_{4}(2) .2, \operatorname{dim} \phi=5, \ell=3$ and $|g|=4,8$;

(27) $G=\mathrm{PSU}_{4}(2) .2, \operatorname{dim} \phi=6, \ell \neq 3$ and $|g|=4,8$;

(28) $G=\mathrm{PSp}_{4}(3), \operatorname{dim} \phi=4$ and $g \in 3 a, 3 b, 3 d$ or $|g|=9$;

(29) $G=\mathrm{PSp}_{4}(3), \operatorname{dim} \phi=5, \ell \neq 2$ and $g \in 3 d$ or $|g|=9$;

(30) $G=\mathrm{PSp}_{4}(3), \operatorname{dim} \phi=6$ and $g \in 3 c$ or $|g|=5,9$;

(31) $G=\operatorname{PSp}_{4}(3) .2$, $\operatorname{dim} \phi=6$ and $|g|=4,8$;

(32) $G=\mathrm{PSU}_{4}(3), \operatorname{dim} \phi=6$ and either $g \in 3 b$ or $|g|=5,7,8,9$;

(33) $G=\mathrm{PSU}_{4}(3) .2_{2}, \operatorname{dim} \phi=6$ and either $g \in 4 d$ or $|g|=8$;

(34) $G=\operatorname{PSU}_{5}(2) .2, \operatorname{dim} \phi=10$ and $|g|=16$; 
(35) $G=\operatorname{Sp}_{4}(2)^{\prime}, \operatorname{dim} \phi=3,4,5$ and $\ell \neq 2,3$;

(36) $G=\operatorname{Sp}_{4}(2)^{\prime}, \operatorname{dim} \phi=6, \ell \neq 2$ and $|g|=5$;

(37) $G=\operatorname{Sp}_{4}(2)^{\prime}, \operatorname{dim} \phi=3,4$ and $\ell=2$;

(38) $G=\operatorname{Sp}_{4}(2)^{\prime}, \operatorname{dim} \phi=2,3,4$ and $\ell=3$;

(39) $G=\mathrm{PSp}_{4}(4), \operatorname{dim} \phi=18$ and $|g|=17$;

(40) $G=\operatorname{PSp}_{6}(2), \operatorname{dim} \phi=7$ and either $g \in 3 a, 4 c$ or $|g|=5,7,8,9$;

(41) $G=\operatorname{PSp}_{6}(2), \operatorname{dim} \phi=8$ and $|g|=7,8,9$;

(42) $G=\mathrm{P} \Omega_{8}^{+}(2), \operatorname{dim} \phi=8$ and either $g \in 3 a, 5 a, 8 b$ or $|g|=7,9$;

(43) $G=\mathrm{PSO}_{8}^{+}(2), \operatorname{dim} \phi=8$ and either $g \in 4 f$ or $|g|=8$.

Moreover, in each of the cases listed above, $\phi(g)$ is indeed almost cyclic.

Proof. Suppose that $L$ is a classical group, and assume furthermore that $L$ is neither orthogonal nor symplectic of even characteristic. By Lemma 5.3, we may assume that $p$ divides $|L|$.

Assume first that $g$ is a semisimple element. Observe that, if $g \notin A_{d}$, then $\phi(g)$ is not almost cyclic, unless $L \in\left\{\mathrm{PSU}_{3}(3), \mathrm{PSU}_{4}(3), \mathrm{PSp}_{4}(3)\right\}$ (see Lemmas 5.4 5.5 5.6. 5.7, 5.8, 5.9 and 5.11). Now, suppose that $g \in A_{d}$. Then (see the argument following Lemma 5.3) either $\phi_{\mid L}$ has an irreducible constituent which is not a Weil representation, or $\phi_{\mid L}$ is an irreducible Weil representation of $L$. It follows that $\phi(g)$ is not almost cyclic, unless either $p>2$ and $L \in\left\{\mathrm{PSL}_{3}(2), \mathrm{PSL}_{3}(4), \mathrm{PSL}_{4}(2)\right\}$ (see Lemmas 5.1 and 5.5), or $L \in\left\{\mathrm{PSU}_{4}(2), \mathrm{PSU}_{4}(3)\right\}$ or $L=\mathrm{PSp}_{4}(3)$ (see Lemmas 5.1 and 5.11).

Next, assume that $g$ is unipotent, without any restriction to $\phi$. Then $\phi(g)$ is not almost cyclic unless $L \in\left\{\mathrm{PSL}_{3}(2), \mathrm{PSL}_{3}(4), \mathrm{PSL}_{4}(2), \mathrm{PSp}_{4}(3), \mathrm{PSU}_{5}(2)\right\}$ (see Lemmas 5.13, 5.14, 5.15 and 5.16). The above listed exceptions are analysed in Section 6. The case $L=\mathrm{PSL}_{3}(2)$ is dealt with in 6.1, yielding items (1) to (6) of the statement. The case $L=\mathrm{PSL}_{3}(4)$ in 6.2 yielding items (7) to (14) of the statement. The case $L=\mathrm{PSL}_{4}(2)$ is dealt with in 6.3 yielding items (15) to (18) of the statement. The case $L=\operatorname{PSU}_{3}(3)$ is dealt with in 6.4, yielding items (19) and (20). The case $L=\mathrm{PSU}_{3}(4)$ is dealt with in 6.5, where it is found that $\phi(g)$ is never almost cyclic, unless $\phi$ is a Weil representation of dimension 12 and $|g|=16$, thus yielding item (21). The case $L=\mathrm{PSU}_{4}(2) \cong \mathrm{PSp}_{4}(3)$ needs some extra care. If we view $L$ as $\mathrm{PSU}_{4}(2)$, the Weil representations of $L$ have dimension 5 and 6 . Thus (see 6.6), we get items (22) to (27). If we view $L$ as $\operatorname{PSp}_{4}(3)$, the Weil representations have dimension 4 and 5. This group is dealt with in Lemma 5.16 if $g$ is unipotent (that is, a 3-element), and in 6.6 if $g$ is semisimple, yielding items (28) to (31). The case $L=\mathrm{PSU}_{4}(3)$ is dealt with in 6.7 yielding items (32) and (33). The case $L=\mathrm{PSU}_{5}(2)$ is dealt with in 5.13 , yielding item (34) of the statement.

Finally, assume that $L$ is a classical group, either orthogonal or symplectic of even characteristic. These groups are dealt with in Section 7. If $L$ is symplectic, then we get items (35) to (41) of the statement, by Lemma 7.1 and Remark 7.2 Lemmas 7.3 and 7.4 deal with the orthogonal groups, yielding items (42) and (43) of the statement.

\section{APPENDIX}

Here some useful routines for MAGMA, kindly provided by the anonymous referee.

function IsAlmostCyclic(A)

$f:=$ CharacteristicPolynomial (A) div MinimalPolynomial (A); 


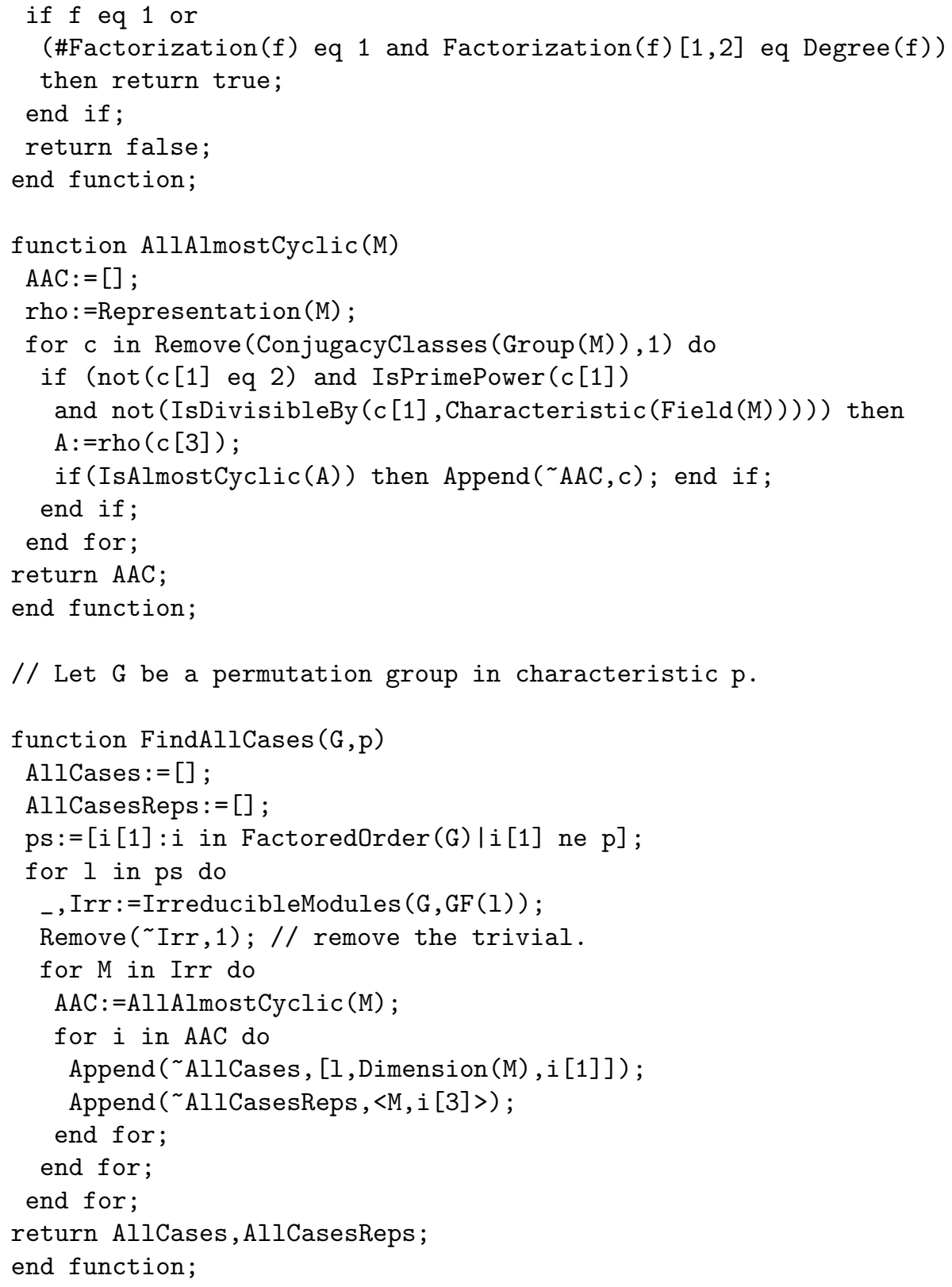

\section{REFERENCES}

[1] J.H. Conway, R.T. Curtis, S.P. Norton, R.A. Parker, R.A. Wilson, Atlas of finite groups. Maximal subgroups and ordinary characters for simple groups, Oxford University Press, Eynsham, 1985.

[2] D. Craven, Groups with a $p$-element acting with a single non-trivial Jordan block on a simple module in characteristic $p$, J. Group Theory 21 (2018), 719-787.

[3] L. Di Martino, M.A. Pellegrini, A.E. Zalesski, On generators and representations of the sporadic simple groups, Comm. Algebra 42 (2014), 880-908. 
[4] L. Di Martino, A.E. Zalesskii, Minimum polynomials and lower bounds for eigenvalue multiplicities of prime-power order elements in representations of classical groups, J. Algebra 243 (2001), 228-263. Corrigendum: J. Algebra 296 (2006), 249-252.

[5] L. Di Martino, A.E. Zalesski, Unipotent elements in representations of finite groups of Lie type, J. Algebra Appl. 11 (2012), 1250038, 25 pp.

[6] L. Di Martino, A.E. Zalesski, Almost cyclic elements in Weil representations of finite classical groups, Comm. Algebra 46 (2018), 2767-2810.

[7] L. Emmett, A.E. Zalesski, On regular orbits of elements of classical groups in their permutation representations, Comm. Algebra 39 (2011), 3356-3409.

[8] The GAP Group, GAP - Groups, Algorithms, and Programming, Version 4.8.8; 2017. (https://www.gap-system.org).

[9] P. Gérardin, Weil representations associated to finite fields, J. Algebra 46 (1977), 54-101.

[10] D. Gorenstein, R. Lyons, R. Solomon, The classification of the finite simple groups. Number 3. Part I. Chapter A. Almost simple K-groups, Mathematical Surveys and Monographs, 40.3. American Mathematical Society, Providence, RI, 1998.

[11] R. Gow, Commutators in finite simple groups of Lie type, Bull. London Math. Soc. 32 (2000), $311-315$.

[12] S. Guest, J. Morris, C.E. Praeger, P. Spiga, On the maximum orders of elements of finite almost simple groups and primitive permutation groups, Trans. Amer. Math. Soc. 367 (2015), $7665-7694$.

[13] R.M. Guralnick, K. Magaard, J. Saxl, P.H. Tiep, Cross characteristic representations of symplectic and unitary groups, J. Algebra 257 (2002), 291-347.

[14] R.M. Guralnick, J. Saxl, Generation of finite almost simple groups by conjugates, J. Algebra 268 (2003), 519-571.

[15] R.M. Guralnick, P.H. Tiep, Low-dimensional representations of special linear groups in cross characteristics, Proc. London Math. Soc. (3) 78 (1999), 116-138.

[16] R.M. Guralnick, P.H. Tiep, Cross characteristic representations of even characteristic symplectic groups, Trans. Amer. Math. Soc. 356 (2004), 4969-5023.

[17] L. Hèthelyi, M. Shőke, A.E. Zalesski, On $p$-stablility in groups and fusion systems, J. Algebra 492 (2017), 253-297.

[18] G. Hiss, G. Malle, Low-dimensional representations of special unitary groups, J. Algebra 236 (2001), 745-767.

[19] C. Hoffman, Cross characteristic projective representations for some classical groups, J. Algebra 229 (2000), 666-677.

[20] C. Jansen, K. Lux, R. Parker, R. Wilson, An atlas of Brauer characters, London Mathematical Society Monographs. New Series, 11. Oxford Science Publications. The Clarendon Press, Oxford University Press, New York, 1995.

[21] W.M. Kantor, Á. Seress, Large element orders and the characteristic of Lie-type simple groups, J. Algebra 322 (2009), 802-832.

[22] P.B. Kleidman, The maximal subgroups of the Steinberg triality groups ${ }^{3} D_{4}(q)$ and of their automorphism groups, J. Algebra 115 (1988), 182-199.

[23] P. Kleidman, M. Liebeck, The subgroup structure of the finite classical groups, London Mathematical Society Lecture Note Series, 129. Cambridge University Press, Cambridge, 1990.

[24] J.B. Olsson, On the blocks of $G L(n, q)$. I, Trans. Amer. Math. Soc. 222 (1976), 143-156.

[25] H. Pollatsek, Irreducible groups generated by transvections over finite fields of characteristic two, J. Algebra 39 (1976), 328-333.

[26] R. Steinberg, Lectures on Chevalley groups, Yale University, New Haven, Conn., 1968.

[27] I.D. Suprunenko, Unipotent elements of nonprime order in representations of classical groups: two large Jordan blocks, Dokl. Nats. Akad. Nauk Belarusi 55 (2011), 21-26, 125.

[28] D.M. Testerman, $A_{1}$-type overgroups of elements of order $p$ in semisimple algebraic groups and the associated finite groups, J. Algebra 177 (1995), 34-76.

[29] D.M. Testerman, A.E. Zalesski, Irreducible representations of simple algebraic groups in which a unipotent element is represented by a matrix with a single non-trivial Jordan block, J. Group Theory 21 (2018), 1-20.

[30] P.H. Tiep, A.E. Zalesskiř, Mod $p$ reducibility of unramified representations of finite groups of Lie type, Proc. London Math. Soc. (3) 84 (2002), 439-472. 
[31] A.V. Vasil'ev, On finite groups isospectral to simple classical groups, J. Algebra 423 (2015), $318-374$

[32] A. Wagner, Determination of the finite primitive reflection groups over an arbitrary field of characteristic not 2. I, Geom. Dedicata 9 (1980), no. 2, 239-253; II, III. Geom. Dedicata 10 (1981), 191-203, 475-523.

[33] H.N. Ward, Representations of symplectic groups, J. Algebra 20 (1972), 182-195.

[34] A.J. Weir, Sylow $p$-subgroups of the classical groups over finite fields with characteristic prime to $p$, Proc. Amer. Math. Soc. 6 (1955), 529-533.

[35] A.E. Zalesski, Minimum polynomials of the elements of prime order in representations of quasi-simple groups, J. Algebra 320 (2008), 2496-2525.

[36] A.E. Zalesskiı̌, V.N. Serežkin, Linear groups generated by transvections, Izv. Akad. Nauk SSSR Ser. Mat. 40 (1976), 26-49, 221.

[37] A.E. Zalesskiı̌, V.N. Serežkin, Finite linear groups generated by reflections, Izv. Akad. Nauk SSSR Ser. Mat. 44 (1980), 1279-1307, 38.

[38] A.V. Zavarnitsin, Recognition of the simple groups $U_{3}(q)$ by element orders, Algebra Logika 45 (2006), 185-202, 252-253; translation in Algebra Logic 45 (2006), 106-116.

E-mail address: lino.dimartino@unimib.it

Dipartimento di Matematica e Applicazioni, Università degli Studi di Milano-Bicocca, Via R. CozZi 55, 20125 Milano, Italy

E-mail address: marcoantonio.pellegrini@unicatt.it

Dipartimento di Matematica e Fisica, Università Cattolica del Sacro Cuore, Via Musei 41, 25121 Brescia, Italy

E-mail address: alexandre.zalesski@gmail.com

Academy of Sciences of Belarus, Minsk, Department of Physics, Mathematics and Informatics, Prospekt Nezalejnasti 66, Minsk, 220000, Belarus 